\title{
THE SLOPES DETERMINED BY $n$ POINTS IN THE PLANE
}

\author{
JEREMY L. MARTIN
}

\begin{abstract}
Let $m_{12}, m_{13}, \ldots, m_{n-1, n}$ be the slopes of the $\left(\begin{array}{c}n \\ 2\end{array}\right)$ lines connecting $n$ points in general position in the plane. The ideal $I_{n}$ of all algebraic relations among the $m_{i j}$ defines a configuration space called the slope variety of the complete graph. We prove that $I_{n}$ is reduced and Cohen-Macaulay, give an explicit Gröbner basis for it, and compute its Hilbert series combinatorially. We proceed chiefly by studying the associated Stanley-Reisner simplicial complex, which has an intricate recursive structure. In addition, we are able to answer many questions about the geometry of the slope variety by translating them into purely combinatorial problems concerning enumeration of trees.
\end{abstract}

\section{INTRODUCTION}

Let there be given $n \geq 2$ distinct points in the plane, connected in pairs by $\left(\begin{array}{l}n \\ 2\end{array}\right)$ lines. The closure of the locus of all slope vectors $\left(m_{12}, \ldots, m_{n-1, n}\right)$ arising from some such configuration is an irreducible algebraic variety of dimension $2 n-3$, called the affine slope variety of the complete graph (as we soon explain). This slope variety turns out to have an unexpectedly rich combinatorial and geometric structure. Our techniques for investigating its properties draw on combinatorics (graph theory and recursive enumeration of trees), commutative algebra (Gröbner bases and Stanley-Reisner theory), and algebraic geometry. Before stating the main theorem, we set it in context by giving an overview of the theory of graph varieties, considered by the author in 10 .

Let $\mathbb{P}^{2}$ be the projective plane over an algebraically closed field $\mathbf{k}$, and let $G$ be a graph with vertices $V$ and edges $E$. A picture $\mathbf{P}$ of $G$ consists of a point $\mathbf{P}(v)$ for each vertex, and a line $\mathbf{P}(e)$ for each edge, subject to the conditions that $\mathbf{P}(v) \in \mathbf{P}(e)$ whenever $v$ is an endpoint of $e$. Thus the data of $n$ points and $\left(\begin{array}{l}n \\ 2\end{array}\right)$ lines described earlier is a picture of the complete graph $K_{n}$ on $n$ vertices.

The set of all pictures of $G$ is called the picture space $\mathcal{X}(G)$. A picture is generic if the points $\mathbf{P}(v)$ are all different; the closure of the locus of generic pictures is called the picture variety $\mathcal{V}(G)$. This is an irreducible component of $\mathcal{X}(G)$ of dimension $2|V|$. Passing to an affine open subset $\tilde{\mathcal{V}}(G) \subset \mathcal{V}(G)$, and projecting onto an affine space $\mathbb{A}_{\mathbf{k}}^{|E|}$ whose coordinates correspond to the slopes of lines $\mathbf{P}(e)$, we obtain the affine slope variety $\tilde{\mathcal{S}}(G)$, of dimension $2|V|-3$.

A rigidity circuit is a graph which admits a decomposition into two spanning trees, and contains no proper subgraph with that property. The most important rigidity circuits are the wheels: a wheel consists of a cycle with an attached central vertex. For each rigidity circuit $C$, there is a corresponding tree polynomial $\tau(C)$, a

2000 Mathematics Subject Classification. 05C10,13P10,14N20.

Key words and phrases. graph, graph variety, slope, Stanley-Reisner ring, shellability, tree, Gröbner basis.

Supported in part by an NSF Postdoctoral Fellowship. 
sum of signed squarefree monomials corresponding to spanning trees appearing in such decompositions; this polynomial is homogeneous and irreducible. The affine slope variety $\tilde{\mathcal{S}}(G)$ is cut out set-theoretically in $\mathbb{A}^{|E|}$ by the polynomials $\tau(C)$, where $C$ ranges over all rigidity circuit subgraphs of $G$. These facts were proven in 10. We can now state the main theorem of this paper.

Theorem 1.1. Let $R_{n}=\mathbf{k}\left[m_{12}, \ldots, m_{n-1, n}\right]$, and let $I_{n}$ be the ideal generated by the tree polynomials of all rigidity circuits in the complete graph $K_{n}$. Then:

(i) The affine slope variety $\tilde{\mathcal{S}}\left(K_{n}\right)$ is defined scheme-theoretically by $I_{n}$. That is, $I_{n}$ is a prime ideal, and $\tilde{\mathcal{S}}\left(K_{n}\right) \cong \operatorname{Spec} R_{n} / I_{n}$.

(ii) The tree polynomials of the wheel subgraphs of $K_{n}$ generate $I_{n}$, and form a Gröbner basis with respect to a certain graded lexicographic order.

(iii) $\tilde{\mathcal{S}}\left(K_{n}\right)$ has dimension $2 n-3$ and degree

$$
\frac{(2 n-4) !}{2^{n-2}(n-2) !}=(2 n-5)(2 n-7) \cdots(3)(1),
$$

the number of perfect matchings on $[1,2 n-4]=\{1,2, \ldots, 2 n-4\}$. Furthermore, the Hilbert series of $R_{n} / I_{n}$ is

$$
\frac{\sum_{k=0}^{n-2} h(n, k) t^{k}}{(1-t)^{2 n-3}},
$$

where $h(n, k)$ counts the number of perfect matchings on $[1,2 n-4]$ with exactly $k$ long pairs, that is, pairs not of the form $\{i, i+1\}$.

(iv) The ring $R_{n} / I_{n}$ and the affine slope variety $\tilde{\mathcal{S}}\left(K_{n}\right)$ are Cohen-Macaulay.

We begin in Section 2 by describing the basic objects - graph varieties and tree polynomials - in somewhat more detail. We do this both to make this paper more self-contained, and because several details of the constructions will be of importance later on. The reader is referred to [10] and 9] for more leisurely treatments of these subjects.

In the first main part of the paper, we construct a monomial ideal $J_{n}$, generated by the initial terms of tree polynomials $\tau(W)$ of wheels $W \subset K_{n}$ with respect to a certain graded lexicographic term ordering. As mentioned previously, the monomials of $\tau(W)$ correspond to coupled spanning trees of $W$, that is, whose complements are also spanning trees. In order to identify the leading term of a wheel polynomial, we need several technical facts about the valences of vertices in coupled trees; these facts comprise Section 3. In Section 4, we introduce the term ordering and, in Theorem 4.3 give a necessary and sufficient combinatorial condition on monomials (somewhat akin to pattern avoidance in permutations) for membership in $J_{n}$.

The second part of the paper consists of Sections 5 . 9 Here we study the StanleyReisner simplicial complex $\Delta(n)$ whose faces correspond to squarefree monomials that do not belong to $J_{n}$. This simplicial complex has a surprising amount of combinatorial structure. First, $\Delta(n)$ is pure, and all its facets may be built up recursively from facets of smaller Stanley-Reisner complexes. Second, this recurrence may be translated into a bijection between facets and a combinatorially more natural set, the binary total partitions, which are enumerated by the double factorial 
numbers (11). These numbers therefore give the degree (or multiplicity) of the ideal $J_{n}$. Third, the description of facets leads to a proof that $\Delta(n)$ is shellable and hence Cohen-Macaulay. This part of the paper (Section 8) is very technical; the arguments are combinatorially elementary but do require careful bookkeeping. The shelling argument leads in turn to a recursive computation of the $h$-vector of $\Delta(n)$; the coefficients of the $h$-vector enumerate perfect matchings by the number of long pairs (a combinatorial problem first considered by Kreweras and Poupard 8]).

At this point, we do not yet know that these results on $\Delta(n)$ correspond to properties of the affine slope variety. The missing piece is to show that $J_{n}$ is not too small-precisely, that it is the initial ideal of an ideal defining $\tilde{\mathcal{S}}\left(K_{n}\right)$ schemetheoretically. As it turns out, it is enough to show that the double factorial numbers give a lower bound for the degree of $\tilde{\mathcal{S}}\left(K_{n}\right)$. We prove this in Sections 10 and 11] Our approach is to consider a nested family of algebraic subsets of $\tilde{\mathcal{S}}\left(K_{n}\right)$ called

flattened slope varieties, whose degree can be bounded from below by a recursive formula (Theorem 10.4). We show that this recurrence is equivalent to one enumerating combinatorial objects called decreasing planar trees, which, like matchings and binary total partitions, are enumerated the double factorials. Using the fact that $J_{n}$ is Cohen-Macaulay and has the appropriate codimension and degree, we conclude that $J_{n}$ is the (complete) initial ideal of $I_{n}$ under an appropriate term ordering; equivalently, the wheel polynomials form a Gröbner basis. The assertions of the main theorem follow more or less immediately.

Together with [10, these results constitute the author's doctoral dissertation [9]. The author thanks his thesis advisor, Mark Haiman, for his ongoing support.

\section{Preliminaries: Graphs and Tree Polynomials}

We assume that the reader is familiar with the elements of graph theory, for which a good general reference is [15. We first fix some notation and terminology. The symbol $\mathbb{N}$ denotes the positive integers. We abbreviate the set $\{m, m+1, \ldots, n\}$ by $[m, n]$.

A graph $G$ is a pair $(V, E)$, where $V=V(G)$ is a finite set of vertices and $E=E(G)$ is a set of edges, or unordered pairs of distinct vertices $e=\{v, w\}$. (Thus we do not allow loops or multiple edges.) For ease of use, we frequently abbreviate $\{v, w\}$ by $v w$. The vertices $v, w$ are the endpoints of $e$. A subgraph of $G$ is a graph $G^{\prime}=\left(V^{\prime}, E^{\prime}\right)$ with $V^{\prime} \subset V$ and $E^{\prime} \subset E$. We use the symbols + and - to denote addition and deletion of edges.

The valence of $v$ with respect to an edge set $E$, written $\operatorname{val}_{E}(v)$, is the number of edges in $E$ incident to $v$. (This is more usually called the degree, but we wish to reserve that term for a different usage.) A vertex of valence 1 is called a leaf. The support of an edge set $E$ is $V(E)=\left\{v \mid \operatorname{val}_{E}(v)>0\right\}$. The complete graph $K_{V}$ is the graph with vertex set $V$ and every two vertices adjacent; thus $\left|E\left(K_{V}\right)\right|=\left(\begin{array}{c}|V| \\ 2\end{array}\right)$. We abbreviate $K_{[1, n]}$ by $K_{n}$. For convenience, we frequently ignore the technical distinction between an edge set $E$ and the graph $(V(E), E)$.

Let $v_{1}, \ldots, v_{k}$ be distinct vertices. The edge set $\left\{v_{1} v_{2}, v_{2} v_{3}, \ldots, v_{k-1} v_{k}\right\}$ is called a path from $v_{1}$ to $v_{k}$, and if $k \geq 3$ then the edge set $\left\{v_{1} v_{2}, v_{2} v_{3}, \ldots, v_{k-1} v_{k}, v_{k} v_{1}\right\}$ is called a cycle or $k$-cycle. It is frequently convenient to describe a path or cycle by listing its vertices in order.

A graph $G$ is connected if every pair of vertices belongs to some common path; it is a tree if it is connected and contains no cycle. Equivalently, a tree may be 
defined as a connected graph with $|E(G)|=|V(G)|-1$, or as a graph in which every pair of vertices belongs to exactly one common path. A spanning tree of $G$ is a tree $T$ with $V(T)=V(G)$ and $E(T) \subset E(G)$. The connected components of a graph are its maximal connected subgraphs.

A graph $G=(V, E)$ is a rigidity pseudocircuit if $E=T \sqcup T^{\prime}$, where $T, T^{\prime}$ are spanning trees and the symbol $\sqcup$ denotes a disjoint union. A rigidity pseudocircuit is a rigidity circuit if it contains no other rigidity pseudocircuit as a proper subgraph. (For the reasons behind this terminology, see [7.) A spanning tree $T \subset E$ is called coupled if its complement is also a spanning tree; we denote the set of coupled trees of $G$ by $\operatorname{Cpl}(G)$.

Let $v_{0}, v_{1}, \ldots, v_{k}$ be distinct vertices. The $k$-wheel $W=W\left(v_{0} ; v_{1}, \ldots, v_{k}\right)$ is defined as the graph with edges $\left\{v_{0} v_{1}, v_{1} v_{2}, \ldots, v_{0} v_{k}\right\} \cup\left\{v_{1} v_{2}, \ldots, v_{k-1} v_{k}, v_{k} v_{1}\right\}$. It is easy to check that every wheel is a rigidity circuit [7, Exercise 4.13]; the figure below shows a 2-tree decomposition of $W\left(v_{0} ; v_{1}, \ldots, v_{6}\right)$.
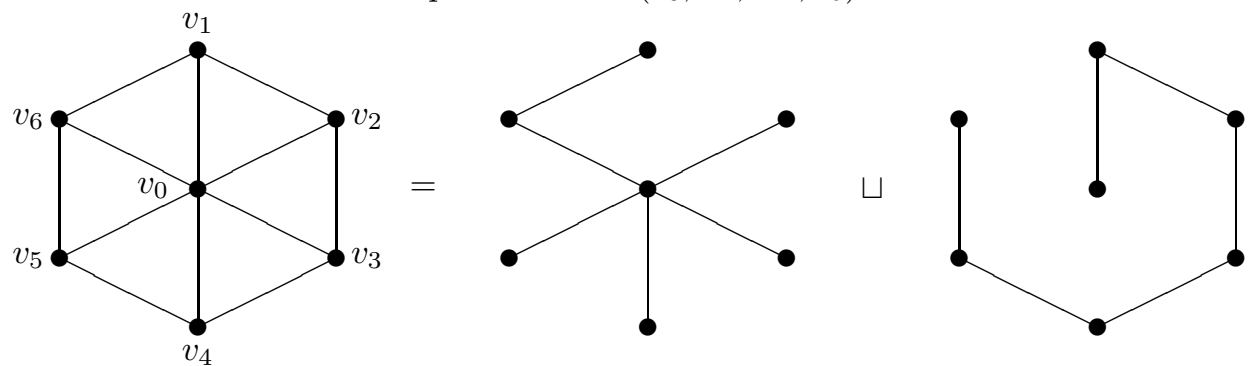

The vertex $v_{0}$ is called the center of $W$, and the vertices $v_{1}, \ldots, v_{k}$ are its spokes. An edge joining two spokes is a chord of the wheel; an edge joining a spoke to the center is a radius. We denote the sets of chords and radii by $\mathrm{Ch}(W)$ and $\operatorname{Rd}(W)$ respectively. It is notationally convenient to set $v_{k+1}=v_{1}$, so that $\mathrm{Ch}(W)=$ $\left\{v_{i} v_{i+1} \mid i \in[1, k]\right\}$.

We now describe certain polynomials associated to rigidity circuits. Let $n \geq 2$ be an integer and $\mathbf{k}$ an algebraically closed field. We will work over the polynomial ring in $\left(\begin{array}{l}n \\ 2\end{array}\right)$ variables

$$
R_{n}=\mathbf{k}\left[m_{12}, \ldots, m_{n-1, n}\right] .
$$

To each edge set $E \subset E\left(K_{n}\right)$ we associate the squarefree monomial

$$
m_{E}=\prod_{e \in E} m_{e} .
$$

For every rigidity circuit $C$ in $K_{n}$, there is an irreducible polynomial, the tree polynomial of $C$, defined up to sign and having the form

$$
\tau(C)=\sum_{T \in \operatorname{Cpl}(C)} \varepsilon(T) m_{T},
$$

where $\varepsilon(T) \in\{1,-1\}$. By [10, Theorem 5.4], the tree polynomial is irreducible and homogeneous of degree $|V(C)|-1=|E(C)| / 2$.

We summarize the construction of $\tau(C)$; for more details and examples, see [10]. For $1 \leq i<j \leq n$, regard the edge $\{i, j\}$ as an oriented edge $(i, j)$, and formally set $(j, i)=-(i, j)$. Fix a spanning tree $T \subset E(C)$ (not necessarily coupled), and let $\bar{T}=E(C) \backslash T$. For every edge $e=v w \in \bar{T}$, the edge set $T+e$ contains a unique cycle $P_{T}(e)$, which we may regard as a set of oriented edges $\left\{(v, w),\left(w, w_{1}\right), \ldots,\left(w_{r}, v\right)\right\}$. 
Let $M$ be the $(|V|-1) \times(|V|-1)$ square matrix with rows indexed by edges $e \in \bar{T}$ and columns indexed by the edges $f \in T$, and whose $(e, f)$ entry is

$$
M_{e, f}= \begin{cases}m_{e}-m_{f} & \text { if } f \in P_{T}(e), \\ m_{f}-m_{e} & \text { if }-f \in P_{T}(e), \\ 0 & \text { otherwise. }\end{cases}
$$

The tree polynomial is then defined as $\tau(C)=\operatorname{det} M$. Up to sign, this construction is independent of the choice of the tree $T$.

The affine slope variety $\tilde{\mathcal{S}}(n)=\tilde{\mathcal{S}}\left(K_{n}\right)$ is defined as follows. Let $p_{1}, \ldots, p_{n}$ be $n$ distinct points in the affine plane $\mathbb{A}_{\mathbf{k}}^{2}$, with no two points lying on the same vertical line. Let $m_{i j} \in \mathbf{k}$ be the slope of the unique line joining $p_{i}$ and $p_{j}$. Thus $\left(m_{12}, \ldots, m_{n-1, n}\right)$ is a point in affine $\left(\begin{array}{l}n \\ 2\end{array}\right)$-space $\mathbb{A}_{\mathbf{k}}^{2}=\operatorname{Spec} R_{n}$, and $\tilde{\mathcal{S}}(n)$ is defined as the closure of the locus of all such points arising from the data $\left(p_{1}, \ldots, p_{n}\right)$.

By [10, Theorem 5.6], the ideal generated by the tree polynomials

$$
\left.I_{n}=\langle\tau(C)| C \subset K_{n} \text { is a rigidity circuit }\right\rangle
$$

cuts out the affine slope variety $\tilde{\mathcal{S}}(n)$ inside Spec $R_{n}$. In fact, $I_{n}$ is generated by the tree polynomials of wheel subgraphs of $K_{n}$. We omit the proof of this fact, since we shall eventually prove the following stronger result: the wheel polynomials form a Gröbner basis for $I_{n}$ with respect to any of a large class of term orderings.

\section{Vertex Valences in Coupled Trees}

This section contains several technical facts concerning the valences of vertices in couples spanning trees of a wheel. These observations lead eventually to an explicit identification of the initial monomial of a wheel polynomial $\tau(W)$ with respect to a certain term ordering.

Throughout this section, we fix a $k$-wheel $W=W\left(v_{0} ; v_{1}, \ldots, v_{k}\right) \subset E\left(K_{n}\right)$. If $T \subset E(W)$ is a coupled spanning tree of $W$, we set $\bar{T}:=E(W) \backslash T$.

For each $i \in[1, k]$ and each coupled tree $T \in \operatorname{Cpl}(W)$, either $\operatorname{val}_{T}\left(v_{i}\right)=1$ or $\operatorname{val}_{T}\left(v_{i}\right)=2$. In addition, not all spokes $v_{i}$ have the same valence, since $T$ is neither $\mathrm{Ch}(W)$ nor $\operatorname{Rd}(W)$. Thus $\mathrm{val}_{T}$ may be regarded as a nonconstant function from $[1, k]$ to $[1,2]$.

Lemma 3.1. Let $T \in \operatorname{Cpl}(W)$ and $i, j \in[1, k]$. Then $\bar{T}$ contains at least one of the following four edges: $v_{0} v_{i}, v_{0} v_{j}, v_{i} v_{i+1}$, and $v_{j-1} v_{j}$.

Proof. Suppose not. Let $i, j$ be a counterexample such that $j-i$ is as small as possible. If necessary, we may reindex the spokes so that $i \leq j$. If $j=i$, then $\operatorname{val}_{T}\left(v_{i}\right)=3$, which is impossible. If $j-i=1$, then $T$ contains the cycle $v_{0}, v_{i}, v_{j}, v_{0}$, and if $j-i=2$, then $T$ contains the cycle $v_{0}, v_{i}, v_{i+1}, v_{j}, v_{0}$.

Now suppose $j-i>2$. Since $T$ contains the path $v_{i+1}, v_{i}, v_{0}, v_{j}, v_{j-1}$, it cannot contain the path $v_{i+1}, v_{i+2}, \ldots, v_{j-1}$. Let $k$ and $\ell$ be the least and greatest indices, respectively, such that $v_{k} v_{k+1} \notin T, v_{\ell-1} v_{\ell} \notin T$, and $i<k \leq \ell<j$. Now $v_{0} v_{k} \notin T$, otherwise $T$ contains the cycle $v_{0}, v_{i}, v_{i+1}, \ldots, v_{k}, v_{0}$. For a similar reason, $v_{0} v_{\ell} \notin T$. But then $k, \ell$ is a counterexample to the lemma, and $|\ell-k|<|j-i|$, which contradicts the choice of $i$ and $j$. 
Lemma 3.2. For each $T \in \operatorname{Cpl}(W)$, at least one of the following conditions is true: either

$$
\text { for all } i \in[1, k], \quad v_{0} v_{i} \in T \text { if and only if } v_{i} v_{i+1} \in \bar{T},
$$

or

$$
\text { for all } i \in[1, k], \quad v_{0} v_{i} \in T \text { if and only if } v_{i-1} v_{i} \in \bar{T} .
$$

Proof. Suppose that both conditions fail. That is, there exists $i \in[1, k]$ such that either $v_{0} v_{i}, v_{i} v_{i+1} \in T$ or $v_{0} v_{i}, v_{i} v_{i+1} \in \bar{T}$. Interchanging $T$ and $\bar{T}$ if necessary, we may assume the former. Moreover, there exists $j \in[1, k]$ such that either $v_{0} v_{j}, v_{j-1} v_{j} \in T$ or $v_{0} v_{j}, v_{j-1} v_{j} \in \bar{T}$. The former is ruled out by Lemma 3.1 so the latter must hold; in particular $i \neq j$.

Since $v_{0} v_{i}, v_{i} v_{i+1} \in T$, the edge $v_{0} v_{i+1}$ must belong to $\bar{T}$. In particular $\bar{T}$ contains the path $v_{j-1}, v_{j}, v_{0}, v_{i+1}$. Thus $\bar{T}$ does not contain the path $v_{i+1}, v_{i+2}, \ldots, v_{j-1}$. Let $h$ be the largest number in $[i+1, j-1]$ such that $v_{h-1} v_{h} \in T$. Since the path $v_{h}, v_{h+1}, \ldots, v_{j}, v_{0}$ is contained in $\bar{T}$, the edge $v_{0} v_{h}$ must belong to $T$. It follows that $T$ contains the edges $v_{0} v_{i}, v_{i} v_{i+1}, v_{h-1} v_{h}$, and $v_{0} v_{h}$, contradicting Lemma 3.1]

Proposition 3.3. Let $d:[1, k] \rightarrow[1,2]$ be a nonconstant function. Then there exist exactly two coupled trees of $W$ for which $\mathrm{val}_{T}=d$.

Proof. To simplify the notation, write $m_{i, j}$ for $m_{v_{i} v_{j}}$. Also, we put $v_{k+1}=v_{1}$. Putting $T=\operatorname{Rd}(W)$ in (4), the matrix $M$ becomes

$$
\left[\begin{array}{ccccc}
m_{0,1}-m_{1,2} & m_{1,2}-m_{0,2} & 0 & \ldots & 0 \\
0 & m_{0,2}-m_{2,3} & m_{2,3}-m_{0,3} & \ldots & 0 \\
\vdots & \vdots & \ddots & & \vdots \\
m_{0, k}-m_{k, 1} & 0 & \ldots & \ldots & m_{k, 1}-m_{0,1}
\end{array}\right]
$$

Taking the determinant, we obtain

$$
\begin{aligned}
\tau(W) & =\prod_{i=1}^{k}\left(m_{0, i}-m_{i, i+1}\right)+(-1)^{k-1} \prod_{i=1}^{k}\left(m_{i, i+1}-m_{0, i+1}\right) \\
& =\prod_{i=1}^{k}\left(m_{0, i}-m_{i, i+1}\right)-\prod_{i=1}^{k}\left(m_{0, i+1}-m_{i, i+1}\right) .
\end{aligned}
$$

The monomial $m_{\mathrm{Ch}(W)}$ appears in both products in ([6), once with coefficient +1 and once with -1 . The same is true for the monomial $m_{\operatorname{Rd}(W)}$. One may easily verify that no other cancellation occurs. Accordingly, to enumerate the number of coupled trees by the valences of spokes, we may substitute $z_{i} z_{i+1}$ for $m_{i, i+1}$ and $z_{i}$ for $m_{0, i}$ in (6) (where the $z_{i}$ are indeterminates) and change all the -'s to +'s. This yields the expression

$$
\prod_{i=1}^{k}\left(z_{i}+z_{i} z_{i+1}\right)+\prod_{i=1}^{k}\left(z_{i+1}+z_{i} z_{i+1}\right)-2\left(\prod_{i=1}^{k} z_{i}+\prod_{i=1}^{k} z_{i}^{2}\right)=2 \sum_{d} z_{i}^{d(i)},
$$

where the sum is taken over all nonconstant functions $d:[1, k] \rightarrow[1,2]$.

Remark 3.4. Proposition 3.3 has the following consequence, which may also be obtained by direct counting: every $k$-wheel has exactly $2^{k+1}-4$ coupled spanning trees. 
Definition 3.5. Let $d:[1, k] \rightarrow[1,2]$ be a nonconstant function. The type of a chord $v_{i} v_{i+1}$ with respect to $d$ is the pair of numbers $d(i), d(i+1)$. The type of a radius $v_{0} v_{i}$ is the pair $d(i-1), d(i+1)$. If $T \in \mathrm{Cpl}(W)$, we define the type of an edge with respect to $T$ to be its type with respect to $d=\operatorname{val}_{T}$. For brevity, we will speak of type-11 chords, type-12 radii, etc.

Lemma 3.6. Let $T \in \operatorname{Cpl}(W)$, and define the types of chords and radii of $W$ with respect to the function $\mathrm{val}_{T}$. Then:

(i) Every type-22 chord belongs to T.

(ii) Every type-11 chord belongs to $\bar{T}$.

(iii) Every type-22 radius belongs to $\bar{T}$.

(iv) Every type-11 radius belongs to $T$.

Proof. Let $v_{i} v_{i+1}$ be a chord. If (i) fails, then the edges $v_{i-1} v_{i}, v_{i+1} v_{i+2}, v_{0} v_{i}$, $v_{0} v_{i+1}$ all belong to $T$. If (ii) fails, then those edges all belong to $\bar{T}$. In either case, Lemma 3.1 is contradicted.

Now let $v_{0} v_{i}$ be a radius Statements (iii) and (iv) are equivalent (switch $T$ and $\bar{T}$ ), so we prove only (iii). If $\operatorname{val}_{T}\left(v_{i}\right)=2$, then $T$ contains the chords $v_{i} v_{i+1}$ and $v_{i-1} v_{i}$ by parts (i) and (ii) of the lemma, so the radius $v_{0} v_{i}$ belongs to $\bar{T}$. On the other hand, if $\operatorname{val}_{T}\left(v_{i}\right)=1$ and $v_{0} v_{i} \in T$, then $\bar{T}$ contains the chords $v_{i} v_{i+1}$ and $v_{i-1} v_{i}$. Since $\operatorname{val}_{T}\left(v_{i+1}\right)=\operatorname{val}_{T}\left(v_{i-1}\right)=2$ by hypothesis, the edges $v_{i-2} v_{i-1}$, $v_{i+1} v_{i+2}, v_{0} v_{i-1}, v_{0} v_{i+1}$ must all belong to $T$, which contradicts Lemma 3.1

Lemma 3.7. Let $T \in \operatorname{Cpl}(W)$ and $1 \leq i<j \leq k$. Suppose that either

$$
\operatorname{val}_{T}\left(v_{i}\right)=1, \quad \operatorname{val}_{T}\left(v_{i+1}\right)=\cdots=\operatorname{val}_{T}\left(v_{j}\right)=2, \quad \operatorname{val}_{T}\left(v_{j+1}\right)=1
$$

or

$$
\operatorname{val}_{T}\left(v_{i}\right)=2, \quad \operatorname{val}_{T}\left(v_{i+1}\right)=\cdots=\operatorname{val}_{T}\left(v_{j}\right)=1, \quad \operatorname{val}_{T}\left(v_{j+1}\right)=2 .
$$

Then exactly one of the two chords $v_{i} v_{i+1}, v_{j} v_{j+1}$ belongs to $T$.

Proof. Suppose that 7ad holds. Then the chords $v_{i+1} v_{i+2}, v_{i+2} v_{i+3}, \ldots, v_{j-1} v_{j}$ all belong to $T$. If both $v_{i} v_{i+1}$ and $v_{j} v_{j+1}$ belong to $T$, then the path $v_{i}, v_{i+1}, \ldots, v_{j+1}$ is a connected component of $T$, which is impossible. On the other hand, if neither of those chords belong to $T$, then $v_{0} v_{i+1}$ and $v_{0} v_{j}$ both belong to $T$. But then $T$ contains the cycle $v_{0}, v_{i+1}, v_{i+2}, \ldots, v_{j}, v_{0}$, which is impossible, If we assume 7b instead of (7a), the same argument goes through, switching 2 with 1 and $T$ with $\bar{T}$.

An alternate formulation of this lemma is as follows. Let a nonconstant function $d:[1, k] \rightarrow[1,2]$ be given, and let $T$ be a coupled tree with $\operatorname{val}_{T}=d$. Traverse the chords of $W$ in order, coloring the type-12 chords (of which there are a positive even number) alternately red and blue. Then either the red chords all belong to $T$ and the blue chords all belong to $\bar{T}$, or vice versa. Moreover, choosing the color of a single type- 12 chord suffices to determine the rest. Having made such a choice, a radius $v_{0} v_{i}$ belongs to $T$ exactly when $d(i)-\left|T \cap\left\{v_{i-1} v_{i}, v_{i} v_{i+1}\right\}\right|=1$.

Alternatively, if the function $d=\mathrm{val}_{T}$ is given, then to determine $T$ uniquely it suffices to specify whether a single type-12 radius $v_{0} v_{i}$ belongs to $T$ or to $\bar{T}$. Without loss of generality $v_{i-1} v_{i}$ is of type 11 or 22 , and $v_{i} v_{i+1}$ is of type 12 . The value of $d(i)$ determines whether or not $v_{i} v_{i+1}$ belongs to $T$, so the rest of $T$ is determined uniquely as in the preceding paragraph. That is: 
Proposition 3.8. Let $T \in \mathrm{Cpl}(W)$. Define the type of each edge in $W$ with respect to $\mathrm{val}_{T}$. Then $T$ contains all type-22 chords, all type-11 radii, half the type-12 chords, in alternation, and a corresponding half of the type-12 radii.

We conclude our technical preliminaries with two results describing the conditions under which a pair of complementary spanning trees may swap edges.

Lemma 3.9. Let $T \in \operatorname{Cpl}(W)$. Suppose that $v_{i-1} v_{i}$ and $v_{i} v_{i+1}$ belong to $\bar{T}$, so that $v_{0} v_{i} \in T$. Assume without loss of generality that the path in $\bar{T}$ from $v_{i}$ to $v_{0}$ passes through $v_{i+1}$. Then $W$ admits the 2-tree decompositions

$$
E_{1}=T-v_{0} v_{i}+v_{i} v_{i+1}, \quad E_{2}=\bar{T}-v_{i} v_{i+1}+v_{0} v_{i}
$$

and

$$
F_{1}=T-v_{0} v_{i-1}+v_{i-1} v_{i}, \quad F_{2}=\bar{T}-v_{i-1} v_{i}+v_{0} v_{i-1} .
$$

Proof. Clearly $E_{1} \sqcup E_{2}=F_{1} \sqcup F_{2}=W$. The edge set $E_{1}$ is a tree because $v_{i}$ is a leaf of $T$, and $E_{2}$ is a tree because $v_{i}$ and $v_{0}$ are in different connected components of $\bar{T}-v_{i} v_{i+1}$. Meanwhile, $F_{1}$ is a tree because $v_{i}$ and $v_{i-1}$ are in different connected components of $T-v_{0} v_{i-1}$, and $F_{2}$ is a tree because $v_{i-1}$ and $v_{0}$ are in different connected components of $\bar{T}-v_{i-1} v_{i}$.

Lemma 3.10. Let $T \in \operatorname{Cpl}(W)$. Suppose that $v_{i-1} v_{i} \in \bar{T}$ and that $v_{0} v_{i}, v_{i} v_{i+1} \in T$, so that $v_{0} v_{i+1} \in \bar{T}$. Then:

(i) If $T$ contains at least one radius other than $v_{0} v_{i}$, then $W$ admits the 2-tree decomposition

$$
E_{1}=T-v_{0} v_{i}+v_{i-1} v_{i}, \quad E_{2}=\bar{T}-v_{i-1} v_{i}+v_{0} v_{i} .
$$

(ii) If $\bar{T}$ contains at least one radius other than $v_{0} v_{i+1}$, then $W$ admits the 2 -tree decomposition

$$
F_{1}=T-v_{i} v_{i+1}+v_{0} v_{i+1}, \quad F_{2}=\bar{T}-v_{0} v_{i+1}+v_{i} v_{i+1} .
$$

Proof. Clearly $W$ is the disjoint union of $E_{1}$ and $E_{2}$ (resp. $F_{1}$ and $F_{2}$ ), so it suffices to show that these edge sets are in fact trees.

(i) $E_{2}$ is a tree because $v_{i}$ is a leaf of $\bar{T}$. If the path from $v_{i-1}$ to $v_{i}$ in $T$ does not go through $v_{0}$, then it must be $\mathrm{Ch}(W)-v_{i-1} v_{i}$. But then $T$ contains at least $k-1$ chords and two radii, which is impossible. Therefore $v_{i-1}$ and $v_{i}$ lie in different connected components of $T-v_{0} v_{i}$, and $E_{1}$ is a tree.

(ii) The path from $v_{0}$ to $v_{i+1}$ in $T$ is just $v_{0}, v_{i}, v_{i+1}$, so $v_{0}$ and $v_{i+1}$ belong to different connected components of $T-v_{i} v_{i+1}$. Hence $F_{1}$ is a tree. If the path from $v_{i}$ to $v_{i+1}$ in $\bar{T}$ does not go through $v_{0}$, then it must be $\mathrm{Ch}(W)-v_{i} v_{i+1}$, which is impossible. So $v_{i}$ and $v_{i+1}$ are in different connected components of $\bar{T}-v_{0} v_{i+1}$. Hence $F_{2}$ is a tree.

\section{The Leading Tree of a Wheel}

The main result of this section is Theorem 4.3 in which we describe explicitly the ideal generated by the initial terms of wheel polynomials. Fix once and for all the following lexicographic order $>$ on the variables $m_{i j}$ :

$$
m_{12}>m_{13}>\ldots>m_{1 n}>m_{23}>\ldots
$$

The corresponding total order for edges of $K_{n}$ is

$$
12>13>\ldots>1 n>23>\ldots
$$


We next extend $>$ to a term ordering on $R_{n}$, graded lexicographic order, which is defined as follows: $\prod_{i, j} m_{i j}^{a_{i j}}>\prod_{i, j} m_{i j}^{b_{i j}}$ if either

$$
\begin{aligned}
& \sum a_{i j}>\sum b_{i j}, \quad \text { or } \\
& \sum a_{i j}=\sum b_{i j} \quad \text { and } \quad a_{k \ell}>b_{k \ell},
\end{aligned}
$$

where $m_{k \ell}$ is the greatest variable (in lexicographic order) such that $a_{k \ell} \neq b_{k \ell}$.

Associating edge sets with square-free monomials as in (2), we may regard the term ordering on $R_{n}$ as defining an extension of the ordering on edges (8) to a total order on subsets of $E\left(K_{n}\right)$. Then (9) becomes the following: for $E, F \subset E\left(K_{n}\right)$, $E>F$ if either $|E|>|F|$, or else $|E|=|F|$ and $\max (E \# F) \in E$, where the symbol \# denotes the symmetric difference operator.

Given a wheel $W \subset E\left(K_{n}\right)$, we wish to identify the leading tree $L T(W)$ of $W$, that is, the coupled tree of $W$ corresponding to the leading monomial of $\tau(W)$ (with respect to graded lex order). We begin by computing the valence of each spoke of $L T(W)$, using the tools developed in the previous section. By Proposition 3.8 this will rule out all but two possibilities for the leading tree.

Proposition 4.1. Let $W=W\left(v_{0} ; v_{1}, \ldots, v_{k}\right)$, with $V=V(W) \subset[1, n]$. Then:

(i) Suppose that $v_{0}=\min (V)$. Then $\operatorname{val}_{L T(W)}\left(v_{0}\right)=k-1$.

(ii) Suppose that $v_{0}=\max (V)$. Then $\operatorname{val}_{L T(W)}\left(v_{0}\right)=1$.

(iii) Suppose that $v_{0} \notin\{\min (V), \max (V)\}$. Then for all $i \in[1, k]$,

$$
\operatorname{val}_{L T(W)}\left(v_{i}\right)= \begin{cases}1 & \text { if } v_{i}>v_{0}, \\ 2 & \text { if } v_{i}<v_{0} .\end{cases}
$$

In particular, if $v_{0}$ is the rth largest member of $V$, where $r \in[2, k]$, then

$$
\operatorname{val}_{L T(W)}\left(v_{0}\right)=r-1 .
$$

Proof. (i,ii) Suppose $v_{0}=\min (V)$. We will show that if $T$ is a coupled tree with $\operatorname{val}_{T}\left(v_{0}\right)<k-1$, then $T$ cannot be the leading tree of $W$. Note that $\bar{T}$ contains at least two radii, say $v_{0} v_{i}$ and $v_{0} v_{j}$. At least one of the chords $v_{i-1} v_{i}, v_{i} v_{i+1}$ belongs to $T$. If both do, then by Lemma 3.9] at least one of

$$
T_{1}=T-v_{i-1} v_{i}+v_{0} v_{i}, \quad T_{2}=T-v_{i} v_{i+1}+v_{0} v_{i}
$$

is coupled. If $v_{i-1} v_{i} \in T$ and $v_{i} v_{i+1} \in \bar{T}$, then $T_{1}$ is coupled by Lemma 3.10 (i). But $T_{1}>T$, so $T \neq L T(W)$ as desired. The proof of (ii) is analogous.

(iii) Suppose that $v_{0}$ is neither the minimum nor the maximum element of $V$. Let $T$ be a coupled tree of $W$ such that $\operatorname{val}_{T}\left(v_{i}\right)=1$ for some $v_{i}<v_{0}$. To show that $T \neq L T(W)$, we will construct a tree $T^{\prime} \in \operatorname{Cpl}(W)$ with $T^{\prime}>T$. There are two cases to consider.

Case 1: $v_{0} v_{i} \in T$. Then $\bar{T}$ contains the chords $v_{i-1} v_{i}$ and $v_{i} v_{i+1}$. Without loss of generality, we may assume that the path from $v_{i}$ to $v_{0}$ in $\bar{T}$ passes through $v_{i+1}$. Then $v_{0} v_{i-1} \in T$. By Lemma 3.9 the tree $T^{\prime}=T-v_{0} v_{i-1}+v_{i-1} v_{i}$ is coupled, and $v_{i-1} v_{i}>v_{0} v_{i-1}$, so $T^{\prime}>T$.

Case 2: $v_{0} v_{i} \in \bar{T}$. Without loss of generality, $v_{i} v_{i+1} \in \bar{T}$ and $v_{i-1} v_{i} \in T$, so $v_{0} v_{i+1} \in T$. Let $T^{\prime}=T-v_{0} v_{i+1}+v_{i} v_{i+1}$. Note that $T^{\prime}>T$. If $T^{\prime}$ is coupled, then we are done. Otherwise, Lemma 3.10 (i) implies that $v_{0} v_{i+1}$ is the unique radius in $T$, that is, $T$ is the path $v_{0}, v_{i+1}, v_{i}, \ldots, v_{i-2}, v_{i-1}$. Since $v_{0} \neq \max (T)$, we may 
choose $j$ such that $v_{j}>v_{0}$; note that $j \neq i$. Then the tree $T^{\prime}=T-v_{j} v_{j+1}+v_{0} v_{j+1}$ is coupled, and $T^{\prime}>T$.

Proposition 4.2. Let $W \subset E\left(K_{n}\right)$ be a $k$-wheel with vertices $V=V(W)$ and center $v_{0}$.

(i) Suppose $v_{0}=\min (V)$. Label the spokes so that $W=W\left(v_{0} ; v_{1}, \ldots, v_{k}\right)$ with $v_{1}=\max \left\{v_{1}, \ldots, v_{k}\right\}$ and $v_{2}>v_{k}$. Then

$$
L T(W)=\operatorname{Rd}(W)-v_{0} v_{1}+v_{k} v_{k+1} .
$$

(ii) Suppose $v_{0}=\max (V)$. Label the spokes so that $W=W\left(v_{0} ; v_{1}, \ldots, v_{k}\right)$ with $v_{1} v_{2}=\min (\mathrm{Ch}(W))$ and $v_{1}>v_{2}$. Then

$$
L T(W)=\mathrm{Ch}(W)-v_{1} v_{2}+v_{0} v_{2} .
$$

Proof. (i) By Proposition 4.1(i), $L T(W)$ contains exactly one chord. Since $v_{0} v_{i}>$ $v_{j} v_{j+1}$ for all $i, j$, the unique radius not in $L T(W)$ must be $\min (\operatorname{Rd}(W))=v_{0} v_{1}$. This implies the desired result because $v_{k} v_{k+1}>v_{1} v_{2}$.

(ii) For each $1 \in[1, k]$, define

$$
T_{i}=\mathrm{Ch}(W)+v_{0} v_{i}-\min \left(v_{i-1} v_{i}, v_{i} v_{i+1}\right) .
$$

By Proposition 4.1 (ii), $L T(W)$ contains exactly one radius, so $L T(W)=T_{i}$ for some $i$. Note that $T_{i}=\mathrm{Ch}(W)+v_{0} v_{i}-v_{1} v_{2}$ for $i=1,2$; in particular $\max \left(T_{1} \# T_{2}\right)=$ $v_{0} v_{2} \in T_{2}$. On the other hand, for $i>2$, we have

$$
\begin{aligned}
\max \left(T_{i} \# T_{2}\right) & =\max \left(v_{0} v_{i}, v_{0} v_{2}, v_{1} v_{2}, \min \left(v_{i-1} v_{i}, v_{i} v_{i+1}\right)\right) \\
& =\min \left(v_{i-1} v_{i}, v_{i} v_{i+1}\right) \in T_{2} .
\end{aligned}
$$

We conclude that $L T(W)=T_{2}$.

In the case that $v_{0} \notin\{\min (V), \max (V)\}$, we have by Proposition 4.1 (iii)

$$
\operatorname{val}_{L T(W)}(i)=\left\{\begin{array}{ll}
1 & \text { if } v_{i}>v_{0} \\
2 & \text { if } v_{i}<v_{0}
\end{array} .\right.
$$

By Proposition 3.3 there are exactly two coupled trees $T, T^{\prime} \in \mathrm{Cpl}(W)$ satisfying these conditions. Moreover

$$
\begin{aligned}
T \cap T^{\prime} & =\{\text { chords of type } 22\} \cup\{\text { radii of type } 11\}, \\
T \# T^{\prime} & =\{\text { chords of type } 12\} \cup\{\text { radii of type } 12\} .
\end{aligned}
$$

Define the critical edge of $W$ to be the maximum element of $T \# T^{\prime}$. Thus $L T(W)$ is whichever of $T, T^{\prime}$ contains the critical edge.

Theorem 4.3. Let $T$ be a tree with $V(T) \subset[1, n]$. Then the following are equivalent:

(i) There exists a wheel $W \subset K_{n}$ such that $T=L T(W)$.

(ii) $T$ contains a path $\left(v_{1}, \ldots, v_{k}\right)$ satisfying the conditions

$$
\begin{aligned}
& k \geq 4, \\
& \max \left(v_{1}, \ldots, v_{k}\right)=v_{1}, \\
& \max \left(v_{2}, \ldots, v_{k}\right)=v_{k}, \\
& v_{2}>v_{k-1} .
\end{aligned}
$$


Proof. The easier direction is (ii) $\Longrightarrow$ (i). Suppose that the path $P=\left(v_{1}, \ldots, v_{k}\right)$ satisfies (10). Consider the wheel $W=W\left(v_{k} ; v_{1}, v_{2}, \ldots, v_{k-1}\right)$. By Proposition 4.1 $L T(W)$ is a path from $v_{k}$ to $v_{1}$. The two possibilities for $L T(W)$ are $P$ and the path

$$
P^{\prime}=\left(v_{k}, v_{2}, v_{3}, \ldots, v_{k-2}, v_{k-1}, v_{1}\right) .
$$

Since $v_{1}>v_{k}>v_{2}>v_{k-1}$, the critical edge of $W$ is

$$
\begin{aligned}
\max \left(P \# P^{\prime}\right) & =\max \left(v_{1} v_{2}, v_{k-1} v_{k}, v_{k} v_{2}, v_{k-1} v_{1}\right) \\
& =v_{k-1} v_{k} \in P
\end{aligned}
$$

so $P=L T(W)$, as desired.

We now show that (i) $\Longrightarrow$ (ii). Let $W=W\left(v_{0} ; v_{1}, \ldots, v_{k}\right)$ with $L T(W)=T$. We will show that $T$ contains a path $P$ satisfying (10).

Case 1: $v_{0}=\min (V)$. Reindex the spokes of $W$ so that $v_{1}=\max \left(v_{1}, \ldots, v_{k}\right)$ and $v_{2}>v_{k}$. By Proposition 4.2 (i) we have

$$
L T(W)=\operatorname{Rd}(W)-v_{0} v_{1}+v_{k} v_{k+1} .
$$

Since $v_{0}<v_{k}<v_{2}<v_{1}$, we may take $P$ to be the path $\left(v_{1}, v_{k}, v_{0}, v_{2}\right)$.

Case 2: $v_{0}=\max (V)$. Reindex the spokes of $W$ so that $v_{1} v_{2}=\min (\mathrm{Ch}(W))$ and $v_{1}>v_{2}$. By Proposition 4.2 (ii) we have

$$
L T(W)=\mathrm{Ch}(W)-v_{1} v_{2}+v_{0} v_{2} .
$$

Let $v_{j}=\max \left(v_{1}, \ldots, v_{k}\right)$. Obviously $j \neq 2$. Also $j \neq 3$ (since $v_{1} v_{2}<v_{2} v_{3}$, implying $\left.v_{1}>v_{3}\right)$. Consider the path $P=\left(v_{0}, v_{2}, v_{3}, \ldots, v_{j}\right)$. The two largest vertices of $P$ are $v_{0}$ and $v_{j}$. If $v_{2}<v_{j-1}$ then $v_{j-1} v_{j}<v_{1} v_{2}$, a contradiction. So $P$ satisfies (10).

Case 3: $v_{0} \notin\{\min (V), \max (V)\}$. Let $e$ be the critical edge of $W$. By definition, $e$ is of type 12 .

Case 3a: The critical edge is a chord $v_{1} v_{2}$. Without loss of generality we may assume $\operatorname{val}_{T}\left(v_{1}\right)=1$ and $\operatorname{val}_{T}\left(v_{2}\right)=2$. Hence $v_{1}>v_{0}>v_{2}$. Now $v_{0} v_{2}>v_{1} v_{2}$, so $v_{0} v_{2}$ is not of type 12 . Hence $v_{0} v_{2}$ is of type 11 and $v_{0} v_{2} \in T$. Then $v_{2} v_{3} \in \bar{T}$, and $d(3)=1$ (otherwise $v_{2} v_{3}$ is of type 22 and $v_{2} v_{3} \in T$, which is not the case). Then $v_{2} v_{3}$ is of type 12 . Since $v_{1} v_{2}$ is the critical edge, we have $v_{1} v_{2}>v_{2} v_{3}$ and $v_{1}<v_{3}$. In particular $v_{1} \neq \max (V)$. Let $v_{j}=\max (V)$, and let $P$ be the path from $v_{1}$ to $v_{j}$ in $T$. If $v_{0} v_{j} \in T$, then $P$ is the path $\left(v_{1}, v_{2}, v_{0}, v_{j}\right)$, which satisfies (10). Otherwise, $P$ is the path

$$
\left(v_{1}, v_{2}, v_{0}, v_{i}, v_{i-1}, \ldots, v_{j}\right)
$$

for some $i$. Therefore $\operatorname{val}_{T}\left(v_{k}\right)=2$ for $i \leq k \leq j-1$, which implies that $v_{j}$ and $v_{1}$ are respectively the largest and second largest vertices of $P$. Moreover, the chord $v_{j+1} v_{j}$ is of type 12, and $v_{1} v_{2}$ is the critical edge. So $v_{j+1}>v_{2}$ and $P$ satisfies (10).

Case 3b: The critical edge is a radius $v_{0} v_{1}$. Without loss of generality we may assume $\operatorname{val}_{T}\left(v_{2}\right)=2$ and $\operatorname{val}_{T}\left(v_{k}\right)=1$. Hence $v_{k}>v_{0}>v_{2}$ and $v_{0} v_{1}<v_{1} v_{2}$. Thus $v_{1} v_{2}$ is not of type 12 and $\operatorname{val}_{T}\left(v_{1}\right)=2$. Let $j$ be the smallest number in $[1, k]$ such that $\operatorname{val}_{T}\left(v_{j}\right)=1$, and let $P$ be the path $\left(v_{0}, v_{1}, \ldots, v_{j}\right)$. All the edges of $P$ other than $v_{0} v_{1}$ and $v_{j-1} v_{j}$ are chords of type 22, thus belong to $T$. So $v_{0} v_{k} \in \bar{T}$ for $2 \leq k \leq j-1$, and $v_{j-1} v_{j} \in T$ (because $\operatorname{val}_{T}\left(v_{j-1}\right)=2$ ). Thus $P \subset T$. Additionally, $j \geq 3$, and the endpoints of $P$, namely $v_{j}$ and $v_{0}$, are respectively its largest and second largest vertex. Finally, $v_{j-1}>v_{1}$ (because $v_{0} v_{1}$, not $v_{0} v_{j-1}$, is the critical edge). Thus $P$ satisfies (10). 
Clearly, the only path on vertices $[1,4]$ satisfying (10) is 4213 . There are two minimal paths on $[1,5]$, namely 53214 and 52314 . (Others, such as 53124, contain subpaths satisfying (10), in this case 3124.) If we let $b(n)$ denote the number of minimal paths on vertices $[1, n]$ satisfying (10), then one can check that the sequence $b(4), b(5), \ldots, b(9)$ is $1,2,5,16,61,272$. This is a subsequence of the Euler numbers or up/down numbers, sequence A000111 in 11.

Many of the results of this section (such as Proposition 4.1) hold for all variable orderings which are "compatible" with the vertices of $K_{n}$, in the sense that if $v, v^{\prime}, v^{\prime \prime} \in[1, n]$ and $v^{\prime}<v^{\prime \prime}$, then $m_{v v^{\prime}}>m_{v v^{\prime \prime}}$. One might also ask about other natural term orderings on $R_{n}$, such as reverse lexicographic order. This is given by $\prod m_{i j}^{a_{i j}}>\prod m_{\text {rlex }}^{b_{i j}}$ if either $\sum a_{i j}>\sum b_{i j}$, or else $\sum a_{i j}=\sum b_{i j}$ and $a_{k \ell}<b_{k \ell}$, where $m_{k \ell}$ is the smallest variable for which $a_{k \ell} \neq b_{k \ell}$ (compare (9)). Then one can show that the leading trees of wheels with respect to reverse lex order are precisely those containing a path $v_{1}, \ldots, v_{k}, k \geq 4$, such that $\max \left(v_{1}, \ldots, v_{k}\right)=v_{1}$, $\max \left(v_{2}, \ldots, v_{k}\right)=v_{k}$, and $v_{2}<v_{k-1}$ (cf. Theorem 4.3).

\section{The Stanley-Reisner Complex}

We begin by recalling the definition of a simplicial complex and some related terminology; for more detail, see for instance [3, ch. 5]. Let $V$ be a finite set of vertices. An (abstract) simplicial complex on $V$ is a set $\Delta$ of subsets of $V$ which contains as members all singleton sets $\{v\}, v \in V$, and with the property that if $F \in \Delta$ and $F^{\prime} \subset F$, then $F^{\prime} \in \Delta$. The elements of $\Delta$ are called faces. A maximal face is called a facet. The dimension of a face $F$ is $\operatorname{dim} F=|F|-1$, and the dimension of $\Delta$ is $\operatorname{dim} \Delta=\max \{\operatorname{dim} F \mid F \in \Delta\}$. A simplicial complex is a simplex if it has exactly one facet, and is pure if all its facets have the same cardinality.

Let $R=\mathbf{k}\left[x_{1}, \ldots, x_{n}\right]$ and let $J \subset R$ be an ideal generated by squarefree monomials. Let $\Delta=\Delta(J)$ be the set of squarefree monomials which do not belong to $J$. Associating each squarefree monomial $x_{j_{1}} \ldots x_{j_{r}}$ with the set $\left\{j_{1}, \ldots, j_{r}\right\}$, we may regard $\Delta$ as a simplicial complex on vertices $[n]$, the Stanley-Reisner complex of $J$.

The Krull dimension of $R / J$ is $1+\operatorname{dim} \Delta$, and the degree of $R / J$ (that is, its multiplicity as an $R$-module) equals the number of facets of $\Delta$. More particularly, the Hilbert series of $R / J$ corresponds to the $h$-vector of $\Delta$, a combinatorial invariant about which we shall have more to say in Section 9

We shall study the monomial ideal $J=J_{n}$ generated by the squarefree monomials corresponding to leading trees of wheels: that is,

$$
J_{n}:=\left(m_{L T(W)} \mid W \subset K_{n} \text { a wheel }\right) \subset R_{n} .
$$

Thus the Stanley-Reisner simplicial complex $\Delta(n)$ of $J_{n}$ is defined by

$$
\Delta(n):=\Delta\left(J_{n}\right)=\left\{E \subset E\left(K_{n}\right) \mid m_{E} \notin J_{n}\right\} .
$$

Slightly more generally, if $V=\left\{v_{1}<\cdots<v_{n}\right\}$ is a finite totally ordered set (typically, $V \subset \mathbb{N}$ ), we define a simplicial complex $\Delta(V)$ on $E\left(K_{V}\right)$, isomorphic to $\Delta(n)$, by replacing the edge $i j$ with $v_{i} v_{j}$.

Note that the vertices of $\Delta(n)$ (in the sense of the definition of a simplicial complex) are the edges of $K_{n}$. By Theorem 4.3 an edge set $E$ is a face of $\Delta(n)$ if and only if $E$ contains no path of the form (10). For this reason, we will say that a path satisfying (10) is forbidden. 
Example 5.1. No path with fewer than three edges satisfies (10), so the complexes $\Delta(2)$ and $\Delta(3)$ are simplices on $E\left(K_{2}\right)$ and $E\left(K_{3}\right)$ respectively. There is a unique forbidden path on four vertices, namely $4213=\{12,13,24\}$, so the facets of $\Delta(4)$ are the edge subsets of $K_{4}$ that omit one edge from the path 4213, namely, $\{13,14,23,24,34\},\{12,14,23,24,34\}$, and $\{12,13,14,23,34\}$. The faces of $\Delta(5)$ are those edge sets containing none of the paths $4213,5213,5214,5314,5324,53214$, or 52314. (These are precisely the paths satisfying (10) which are minimal under inclusion; see the discussion following the proof of Theorem 4.3)

The main result on the structure of facets of $\Delta(n)$ is as follows.

Theorem 5.2. Let $n \geq 3$, and let $\Delta(n)$ be the Stanley-Reisner simplicial complex just described. Then:

(i) $\Delta(n)$ is pure of codimension $2 n-4$. Moreover, every facet $F \in \Delta(n)$ is 2-connected, and may be written uniquely as a disjoint union

$$
F=F^{1} \sqcup F^{2} \sqcup\{1 n\}
$$

with the following properties: each $F^{i}$ is a facet of the complex $\Delta\left(V\left(F^{i}\right)\right)$, and

$$
\begin{aligned}
& V\left(F^{1}\right) \cup V\left(F^{2}\right)=[1, n], \\
& V\left(F^{1}\right) \cap V\left(F^{2}\right)=\left\{\max \left(V\left(F^{1}\right)\right)\right\}, \\
& 1 \in V\left(F^{1}\right), \\
& n \in V\left(F^{2}\right) .
\end{aligned}
$$

(ii) Let $F^{1}, F^{2}$ be facets of the complexes $\Delta\left(V\left(F^{1}\right)\right)$ and $\Delta\left(V\left(F^{2}\right)\right)$ respectively, satisfying the four conditions just given. Then the edge set $F^{1} \sqcup F^{2} \sqcup\{1 n\}$ is a facet of $\Delta(n)$.

We will need several facts about connectivity and 2-connectivity of graphs, which we summarize here; for details see Chapter 4 of [15. Let $G=(V, E)$ be a connected graph, and denote by $G-v$ the graph obtained by deleting a vertex $v$. If $G-v$ is disconnected, then $v$ is called a cut-vertex of $G$ (or of $E$ ). The vertex $v$ separates the vertices $w$ and $x$ if $w$ and $x$ lie in different connected components of $G-v$. Equivalently, $v$ lies on every path between $w$ and $x$. $G$ is called 2-connected if it has no cut-vertex. (In particular, we consider $K_{2}$ to be 2 -connected, which is not the usual convention.)

A block of $G$ is a maximal 2-connected subgraph of $G$. Every edge of $G$ belongs to exactly one block. A vertex belongs to more than one block if and only if it is a cut-vertex; in addition, two blocks share at most one vertex. In particular, if $G^{\prime}=\left(V^{\prime}, E^{\prime}\right)$ is a block of $G, v \in V^{\prime}$ is not a cut-vertex of $G$, and $w \in V \backslash V^{\prime}$, then there is a unique vertex $x \in V^{\prime}$ such that $x$ is a cut-vertex of $G$ separating $v$ and $w$.

If $v$ is a cut-vertex of a connected graph $G$, then a $v$-lobe of $G$ is a maximal connected subgraph not having $v$ as a cut-vertex. Note that this decomposition is less fine than the block decomposition of $G$ : for instance, if $G$ is a path $v_{1}, v_{2}, \ldots, v_{r}$, then each edge is a block, but there are only two lobes with respect to each cutvertex.

Finally, we will need the following special case of Menger's Theorem [15, p. 167]. Let $v, w$ be nonadjacent vertices of a graph $G$. Then exactly one of the following 
conditions is true: either $G$ contains a cut-vertex separating $v$ and $w$, or else $v, w$ lie on a common cycle $C$ in $G$.

We now start our investigation of the simplicial complexes $\Delta(n)$. As noted in Example 5.1] $\Delta(2)$ and $\Delta(3)$ are simplices; in particular, $\Delta(n)$ is pure of dimension $2 n-4$ for $n \leq 3$. For $n>3$, the following criterion for nonmembership in $\Delta(n)$ will be very useful.

Lemma 5.3. Let $C$ be a cycle on vertices $V \subset[1, n]$. If $\min (V)$ and $\max (V)$ are not adjacent in $C$, then $C$ contains a forbidden path.

Proof. We proceed by induction on $|V|$. Without loss of generality we may assume $V=[1, n]$. If $n=3$, the statement is vacuously true. If $n=4$, then the only possibility is $C=1,3,4,2,1$, which contains the forbidden path $4,2,1,3$.

Now suppose that $n>4$, and the vertices $1, n$ are not adjacent. If $C$ contains the edge $\{1, n-1\}$, then it contains a forbidden path of the form $n-1,1, \ldots, n$. Otherwise, label the vertices of $C$ in order as $v_{1}, \ldots, v_{n}, v_{1}$, with $v_{1}=n, v_{i}=n-1$, and $v_{j}=1$. In particular, $1<i+1<j<n$.

Let $r=n-i+1$, so that $n>r \geq 4$. By induction, the $r$-cycle $v_{i}, v_{i+1}, \ldots, v_{n}, v_{i}$ contains a forbidden path $P$. If $n-1$ and $v_{n}$ are not adjacent in $P$, then $P \subset C$ and we are done. Otherwise, $n-1$ is an endpoint of $P$, with unique neighbor $v_{n}$. Let $P^{\prime}$ be the path obtained from $P$ by deleting the edge $\left\{n-1, v_{n}\right\}$ and replacing it with $\left\{n, v_{n}\right\}$. Then $P^{\prime}$ is also forbidden, and $P^{\prime} \subset C$ as desired.

Corollary 5.4. Let $F$ be a 2-connected face of $\Delta(n)$, and let $V=V(F)$. Then the edge $\{\min (V), \max (V)\}$ belongs to $F$.

Proof. Since $F$ is 2-connected, it contains a cycle $C$ supported at both $\min (V)$ and $\max (V)$. By Lemma 5.3 these vertices are adjacent in $C$, hence in $F$.

By Theorem 4.3 no forbidden path in $K_{n}$ contains either of the edges $\{1, n\}$ or $\{n-1, n\}$. Therefore, both of these edges belong to every facet of $\Delta(n)$. We will frequently work with the face $\hat{F}=F \backslash\{\{1, n\}\}$. Note that $\hat{F}$ is a face of $\Delta(n)$ of cardinality $|F|-1$.

Lemma 5.5. Let $n \geq 3$, and let $F$ be a facet of $\Delta(n)$. Then both $F$ and $\hat{F}$ are connected. However, $\hat{F}$ is not 2-connected; in particular, $\hat{F}$ has a cut-vertex separating 1 and $n$.

Proof. Suppose that $F$ is disconnected. Let $e$ be an edge whose endpoints are the largest vertices of the connected components of $F$ to which they belong. Then it is easy to check that $F+e \in \Delta(n)$, which contradicts the hypothesis that $F$ is a facet.

Suppose that $\hat{F}$ is disconnected. Let $H$ be the connected component of $\hat{F}$ containing the edge $\{n-1, n\}$. Then the vertex 1 does not belong to $H$; in particular, $e=\{1, n-1\} \notin F$. Let $F^{\prime}=\hat{F}+e$. Either $F^{\prime}$ contains a forbidden path, or it is a face of $\Delta(n)$. If $F^{\prime}$ contains a forbidden path $P$, then $P$ contains $e$, hence must be of the form $n-1,1, \ldots, v, n$. But then $P-e \subset F$, which is impossible. On the other hand, if $F^{\prime} \in \Delta(n)$, then $F^{\prime}+\{1, n\}=F+e \in \Delta(n)$, which contradicts the hypothesis that $F$ is a facet. We conclude that $\hat{F}$ is connected. However, the vertices 1 and $n$ are not adjacent in $\hat{F}$, so by Lemma 5.3 they cannot lie in a common cycle. The last assertion of this lemma now follows from the special case of Menger's Theorem mentioned above. 
Lemma 5.6. Let $n \geq 3$, and let $F$ be a facet of $\Delta(n)$. Then the vertex 1 is not a cut-vertex of $\hat{F}=F-\{1, n\}$.

Proof. We prove the contrapositive. Suppose that 1 is a cut-vertex of $\hat{F}$. Let $A$ be the lobe containing vertex $n$, and let $B=F \backslash A$. Then neither $A$ nor $B$ is empty, and their only common vertex is 1 . Let

$$
\begin{aligned}
& x=\max (V(B)), \\
& y=\min \{v \in V(A) \mid 1 v \in \hat{F}\} .
\end{aligned}
$$

Since $x \notin V(A)$, we have $x \neq y$. We consider the cases $x<y$ and $x>y$ separately. In both cases, the method of proof is to construct a forbidden path in $\hat{F}$, which is a contradiction because $\hat{F} \in \Delta(n)$.

Case 1: $x<y$. Suppose that $\hat{F}+x y$ contains a forbidden path $P$. Then $x y \in P$, since $\hat{F} \in \Delta(n)$ and $P \not \subset \hat{F}$. Label the vertices of $P$ in order as $a, \ldots, x, y, \ldots, b$. Then $a, b \geq y>x$, so $a, b \in V(A)$. Hence the subpath of $P$ from $a$ to $x$ must go through the vertex 1 . Therefore $P$ decomposes as $P_{1} \sqcup P_{2} \sqcup\{x y\} \sqcup P_{3}$, where $P_{1}$ is a path from $a$ to $1, P_{2}$ is a path from 1 to $x$, and $P_{3}$ is a path from $y$ to $b$. But then $P_{1} \sqcup\{1 y\} \sqcup P_{3}$ is a forbidden path in $\hat{F}$. It follows that no such path $P$ exists, and $\hat{F}+x y \in \Delta(n)$ But then so $F+x y$ is also an element of $\Delta(n)$, which contradicts the hypothesis that $F$ is a facet of $\Delta(n)$.

Case 2: $x>y$. The face $\hat{F}$ contains a path $y, 1, x_{1}, \ldots, x_{r}=x$; call this $P$. If $x_{1}<y$, then the subpath of $P$ from $y$ to $x_{i}$ is forbidden, where $i$ is the smallest index such that $x_{i}>y$; this is impossible. Therefore $x_{1}>y$. On the other hand, $A$ contains a path $P^{\prime}$ of the form

$$
y=y_{1}, y_{2}, \ldots, y_{s}=n
$$

with $y_{i} \neq 1$ for all $i$ (because 1 is not a cut-vertex of $A$ ). Let $t$ be the smallest number such that $y_{t}>x_{1}$. Then $\hat{F}$ contains the forbidden path

$$
x_{1}, 1, y=y_{1}, y_{2}, \ldots, y_{t},
$$

which is a contradiction.

By this lemma and the remarks following the statement of Theorem 5.2 each facet $F$ has a unique vertex $a=a(F)$ with the following properties: $a$ is a cut-vertex of $\hat{F}$ separating 1 and $n$, and $a$ and 1 belong to the same 2-connected block of $\hat{F}$.

Lemma 5.7. Let $F$ be a facet of $\Delta(n)$, and let $a=a(F)$ be the vertex just described. Let $F^{1}$ be the a-lobe of $\hat{F}$ containing 1 , and let $F^{2}=F \backslash F^{1}$ be the union of all other a-lobes. Then $a=\max \left(V\left(F^{1}\right)\right)$.

Proof. We first prove a weaker statement. Let $H$ be the unique block of $F$ containing 1 (and thus $a$ ); we will show that $a=\max (V(H))$. Suppose not, and let $m=\max (V(H))>a$. Then $H$ contains the edge $1 m$ by Corollary [5.4 Since $H$ is connected, it contains a path $P_{1}$ from 1 to $a$ which does not go through $m$. Moreover, $F^{2}$ contains a path from $a$ to $n$, which we can truncate at the first vertex $v>m$ to obtain a path $P_{2}$. Then $\{1 m\} \sqcup P_{1} \sqcup P_{2}$ is a forbidden path in $F$ from $m$ to $v$. This is a contradiction, so $a=m$.

We now prove the full lemma. Suppose that $m=\max \left(V\left(F^{1}\right)\right)>a$. By the weaker case, $m \notin V(H)$, so there is a unique cut-vertex $b \in V(H)$ separating 1 and $m$. Then $b \neq a$ (otherwise $m=n \in V\left(F^{1}\right)$, which contradicts the definition 
of $F^{1}$ ) and $b \neq 1$ (by Lemma [5.6). Therefore $1<b<a<m$. Then $1 a \in H$ by Corollary 5.4 Moreover, $H$ contains a path $P$ from 1 to $b$ which does not go through $a$. Additionally, $F^{1} \backslash H$ contains a path from $b$ to $m$, which we truncate at the first vertex greater than $a$ to obtain a path $P^{\prime}$. Then $F$ contains a forbidden path, namely $\{1 a\} \sqcup P \sqcup P^{\prime}$; this is a contradiction.

With all of these technical results in hand, we now proceed to the proof of the main theorem characterizing facets of the Stanley-Reisner complex $\Delta(n)$.

Proof of Theorem 5.2 Let $a, F^{1}$ and $F^{2}$ be as defined in Lemma 5.7 To complete the proof of the theorem, we must show that each $F^{i}$ is 2-connected and is a facet of the complex $\Delta\left(V\left(F^{i}\right)\right)$, and that the decomposition is unique. This last assertion is equivalent to the statement that $a$ is the only cut-vertex of $\hat{F}$.

Suppose that $F^{1}$ is not a facet of $\Delta\left(V\left(F^{1}\right)\right)$. That is, there is some edge $e \notin F^{1}$ such that $F^{1} \cup\{e\} \in \Delta\left(V\left(F^{1}\right)\right)$. Note that $e \notin F$. Since $F$ is a facet of $\Delta(n)$, the edge set $F \cup\{e\}$ must contain a forbidden path $P$. Indeed, $P \subset \hat{F} \cup\{e\}$, since no forbidden path contains the edge $\{1, n\}$. On the other hand, $P \not \subset F^{1} \cup\{e\}$ and $P \not \subset F^{2}$, since both $F^{1} \cup\{e\}$ and $F^{2}$ are faces of $\Delta(n)$. Since both these edge sets are connected, it follows that $P$ must go through $a$ and have an endpoint $b \in V\left(F^{1}\right) \backslash\{a\}$. But by Lemma 5.7 the endpoints of $P$ are not its two greatest vertices. Therefore $P$ is not forbidden, and no such $e$ exists. It follows that $F^{1}$ is a facet of $\Delta\left(V\left(F^{1}\right)\right)$, as desired. The same argument shows that $F^{2}$ must be a facet of $\Delta\left(V\left(F^{2}\right)\right)$.

We now show by induction on $n$ that $F$ is 2 -connected and has cardinality $2 n-3$, and that $a$ is the only cut-vertex of $\hat{F}$. The base case $n=3$ is easy: the only facet of $\Delta(3)$ is $F=\{12,13,23\}$. This has the right cardinality and is 2-connected, and $F-13$ has a unique cut-vertex, namely 2. Now suppose $n>3$. By induction $F^{1}$ and $F^{2}$ are 2-connected, and they share the vertex $a$, so $a$ is the only cut-vertex of $\hat{F}$. Furthermore, $F^{1}$ and $F^{2}$ are precisely the 2-connected components of $\hat{F}$. Since $F=\hat{F}+1 n$, and this edge has one endpoint in each of $V\left(F^{1}\right) \backslash\{a\}$ and $V\left(F^{2}\right) \backslash\{a\}$, it follows that $F$ is 2-connected. Also, $\left|V\left(F^{2}\right)\right|=n-\left|V\left(F^{1}\right)\right|-1$, so by induction

$$
|F|=1+\left(2\left|V\left(F^{1}\right)\right|-3\right)+\left(2 n-2\left|V\left(F^{1}\right)\right|-1\right)=2 n-3 .
$$

Finally, suppose that $F^{1}$ and $F^{2}$ are facets of $\Delta\left(V\left(F^{1}\right)\right)$ and $\Delta\left(V\left(F^{2}\right)\right)$, respectively, and that the vertex sets $V\left(F^{i}\right)$ satisfy the conditions (13). So $a=$ $\max \left(V\left(F^{1}\right)\right)$ is a cut-vertex of $F^{1} \cup F^{2}$. Suppose that $F$ contains a forbidden path $P$. Note that $1 n \notin P$ and neither $F^{1}$ nor $F^{2}$ contains $P$ as a subset, so $P$ must have an endpoint in $V\left(F^{1}\right) \backslash\{a\}$, which contradicts the condition $a=\max \left(V\left(F^{1}\right)\right)$. Therefore $F \in \Delta(n)$. Moreover, $|F|=2 n-3$ by (14), so $F$ is a facet.

Example 5.8. The edge set $F$ shown below is a facet of the complex $\Delta(6)$; this can be checked routinely using the characterization of forbidden paths in Theorem 4.3 . The subsets $F^{1}$ and $F^{2}$ are shown as well. Note that 4 is the unique cut-vertex of $\hat{F}=F-16$, and that the corresponding lobes are exactly $F^{1}$ and $F^{2}$. 

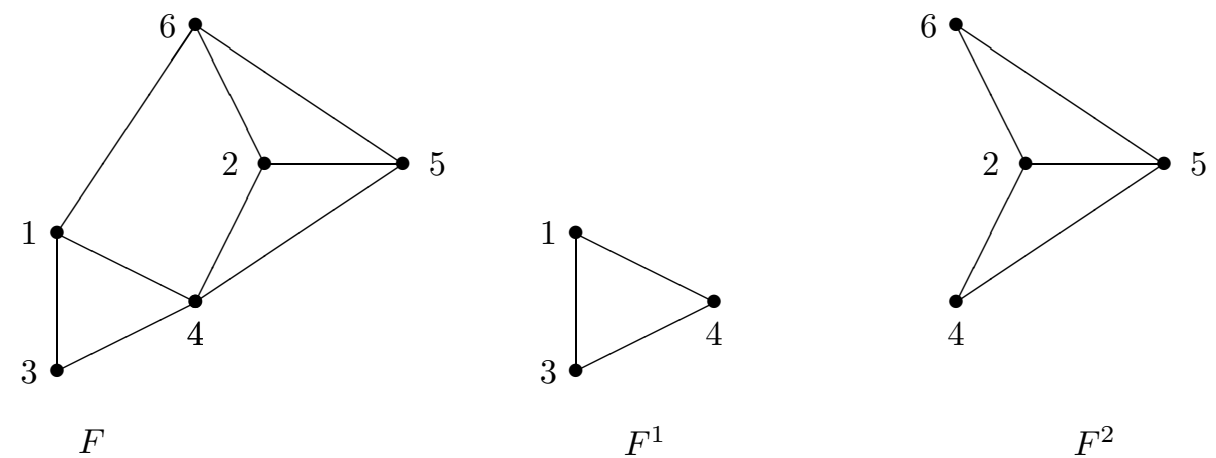

\section{Representing Facets as Binary Trees}

Let $F \in \Delta(n)$ be a facet. Iterating the decomposition of Theorem [5.2 we can construct a binary tree corresponding to $F$. In this section, we characterize these trees explicitly. Where no confusion can arise, we abbreviate sets of one-digit integers by a single word, e.g., $13467=\{1,3,4,6,7\}$.

We begin by reviewing some general facts about planar trees. A rooted tree is a tree $T=(V, E)$ with a distinguished vertex $\operatorname{rt}(T)$, the root of $T$. We refer to the vertices of a rooted tree as nodes; this is in order to avoid confusion when the nodes are labeled with sets of vertices of $K_{n}$. We sometimes abuse notation by writing $v \in T$ instead of $v \in V$. If $v, w$ are distinct nodes of $T$, we say that $v$ is an ancestor of $w$ (equivalently, $w$ is a descendant of $v$ ) if $v$ lies on the unique path from $\operatorname{rt}(T)$ to $w$. If $v$ is an ancestor of $w$ and $v w \in E$, then $v$ is the parent of $w$ and $w$ is a child of $v$. In this case we write $v=v^{(P)}$. Two vertices with the same parent are called siblings. We denote by $T \mid v$ the subtree of $T$ consisting of the node $v$ and all its descendants.

A rooted planar tree is a rooted tree in which, for every node $v \in V$, the set of children of $v$ is equipped with a total ordering, the birth ordering. That is, we can say which of two siblings (or two subtrees whose roots are siblings) is older than the other. We denote the $i$ th oldest child of $v$ by $v^{(i)}$. This notation can be iterated: for instance, $v^{(22)}$ means the second child of the second child of $v$. A node $w$ is said to be firstborn if it is the oldest child of its parent; that is, $w=w^{(P 1)}$. If $v=\operatorname{rt}(T)^{\left(j_{1} \ldots j_{s}\right)}$, then we call the sequence of numbers $\left(j_{i}\right)$ the pedigree of $v$. A binary tree is a rooted planar tree in which each node has either zero or two children. The traversal $\operatorname{trav}(T)$ of a rooted planar tree $T$ is the list of nodes of $T$ in the following order: first $\operatorname{rt}(T)$, then the nodes of $T^{(1)}$ in traversal order, then the nodes of $T^{(2)}$ in traversal order, and so on. (This is the order in which the nodes would be visited in the course of a depth-first search.)

Definition 6.1. Let $F \in \Delta(V)$ be a facet. Define a binary tree $\mathbf{T}(F)$ recursively as follows. If $|V|=2$, then $\mathbf{T}(F)$ has one node, labeled by $V$ itself. Otherwise, $\mathbf{T}(F)$ is the binary tree with root $V$, older subtree $\mathbf{T}\left(F^{1}\right)$, and younger subtree $\mathbf{T}\left(F^{2}\right)$, where $F^{1}, F^{2}$ are as in Theorem 5.2 $\mathbf{T}(F)$ is called the decomposition tree of $F$.

Example 6.2. Let $F$ be the facet shown in Example 5.8 The decomposition tree of $F$ has root node 123456, and by our computation of $F^{1}$ and $F^{2}$, the children of the root are 134 and 2456 . Continuing in this way, we may calculate the complete decomposition tree, which is as follows: 


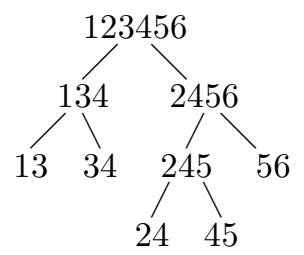

There is a bijection $\mathbf{E}$ from nodes $X$ of $\mathbf{T}(F)$ to edges of $F$, given by

$$
\mathbf{E}(X)=\{\min (X), \max (X)\} .
$$

We also write $\mathbf{E}(T)=\{\mathbf{E}(X) \mid X \in T\}$, where $T$ is a tree or a set of nodes. The construction of $\mathbf{T}(F)$ and Theorem 5.2 immediately yield the following facts about decomposition trees.

Proposition 6.3. Let $\mathbf{T}(F)$ be the decomposition tree of a facet $F \in \Delta(V)$. Then each node is labeled with at least two vertices of $V$ (for short, $|X| \geq 2$ ), and the leaves of $\mathbf{T}(F)$ are exactly those nodes $X$ for which $|X|=2$. Each node $X$ that is not a leaf satisfies the following conditions:

$$
\begin{aligned}
& \text { (a) } \min (X) \in X^{(1)} \backslash X^{(2)} \\
& \text { (b) } \max (X) \in X^{(2)} \backslash X^{(1)} \\
& \text { (c) } X^{(1)} \cup X^{(2)}=X \\
& \text { (d) } X^{(1)} \cap X^{(2)}=\left\{\max \left(X^{(1)}\right)\right\}
\end{aligned}
$$

A tree satisfying these conditions will be called admissible.

Theorem 6.4. Let $V \subset \mathbb{N},|V| \geq 2$. Let $\operatorname{Adm}(V)$ be the set of all admissible binary trees with root $V$. Then the function $\mathbf{T}$ is a bijection from facets of $\Delta(V)$ to $\operatorname{Adm}(V)$. Moreover, the functions $\mathbf{T}$ and $\mathbf{E}$ are inverses.

Proof. Note that $\mathbf{E}(T) \in \Delta(V)$ for all $T \in \operatorname{Adm}(V)$, by Theorem 5.2 Next, we show that the function $\mathbf{E}$ is injective on $\operatorname{Adm}(V)$. Let $T \neq T^{\prime}$ be admissible trees with the same root $V$. Then there are nodes $X, X^{\prime}$ of $T, T^{\prime}$ respectively which have the same pedigree but different labels. Choose these two nodes as close to the root as possible, so that their parents have the same label $Y$. Then the edge sets $E-\mathbf{E}(Y)$ and $E^{\prime}-\mathbf{E}(Y)$ belong to different blocks, so the facets $E=\mathbf{E}(T \mid Y)$ and $E^{\prime}=\mathbf{E}\left(T^{\prime} \mid Y\right)$ are distinct and $\mathbf{E}(T) \neq \mathbf{E}\left(T^{\prime}\right)$ as desired. It is clear from the definitions that $\mathbf{E}(\mathbf{T}(F))=F$ for every facet $F$, so we are done.

We may now speak of a decomposition tree on $V$ as shorthand for a decomposition tree of facets of $\Delta(V)$.

Remark 6.5. It follows from Proposition 6.3 that for each parent node $X$ in a decomposition tree, one has $X^{(2)}=\left(X \backslash X^{(1)}\right) \cup\left\{\max \left(X^{(1)}\right)\right\}$. In particular, each older child determines its younger sibling uniquely, a fact that will be useful later.

\section{Binary Total Partitions}

By Theorem 6.4 the degree of the ideal $J_{n}$ is the number of decomposition trees with root $V$. However, if we try to calculate this number directly using the conditions of Proposition 6.3. we wind up with an awkward recursive formula with no apparent closed-form solution. Instead, we construct a bijection from $\operatorname{Adm}(V)$ to a more easily enumerated set, the binary total partitions of $V$. We begin by defining these trees and listing some salient properties. 
Definition 7.1. Let $V \subset \mathbb{N}$. A binary total partition of $V$ is a binary tree $T$ with nodes labeled by nonempty subsets of $V$, such that

- $\operatorname{rt}(T)=V$;

- If $X$ is a leaf, then $|X|=1$; and

- If $X$ has children, then it is their disjoint union, and $\max (X) \in X^{(2)}$.

It would be simpler to define binary total partitions by ignoring the distinction between the two children of a given parent. However, the bijection between decomposition trees and binary total partitions will be easier to describe if we adopt the convention that each parent and its younger child have a common maximum.

The set of all binary total partitions of $V$ is denoted $\operatorname{BPP}(V)$. It is elementary to show that

$$
|\operatorname{BPP}(V)|=\frac{(2 n-4) !}{2^{n-2}(n-2) !}=(2 n-5)(2 n-7) \cdots(3)(1) .
$$

where $n=|V|+1$ [13, Example 5.2.6].

For $T \in \operatorname{BPP}(V)$ and $j \notin V$, define $\operatorname{Aug}_{j}(T)$ to be the tree obtained by adding the element $j$ to the root of $T$ (augmenting $T$ by $j$ ). Clearly $\operatorname{Aug}_{j}$ is a bijection from $\mathrm{BPP}(V)$ to $\operatorname{BPP}(V, j)=\left\{\operatorname{Aug}_{j}(T) \mid T \in \operatorname{BPP}(V)\right\}$. We will say that $T \in \operatorname{BPP}(V, j)$ is proper if $j<\min (V)$.

Let $S \subset \mathbb{N}, j \notin S, j^{\prime}=\min (S \cup\{j\})$, and $S^{\prime}=S \cup\{j\} \backslash\left\{j^{\prime}\right\}$. Define a straightening map $\psi_{j j^{\prime}}$ on trees $T \in \operatorname{BPP}(S, j)$ as follows. For each $k \in S$ with $k<$ $j$, replace all occurrences of $k$ in labels of non-root nodes of $T$ with the next largest member of $S \cup\{j\}$. Note that $\psi_{j j^{\prime}}$ is a bijection from $\operatorname{BPP}(S, j)$ to $\operatorname{BPP}\left(S^{\prime}, j^{\prime}\right)$; in particular, $\psi_{j j}$ is the identity map. In what follows, it is frequently convenient and unambiguous to write simply $\psi$ instead of $\psi_{j j^{\prime}}$; it is only necessary to specify the subscripts if we want to work with the inverse function.

Example 7.2. Let $S=\{1,2,4,5\}$ and $j=3$. Put $j^{\prime}=\min (S \cup\{j\})=1$ and $S^{\prime}=S \cup\{j\} \backslash\left\{j^{\prime}\right\}=\{2,3,4,5\}$. The following figure shows a tree $T \in \operatorname{BPP}(S, j)$ and the tree $\psi_{j j^{\prime}}(T) \in \operatorname{BPP}\left(S^{\prime}, j^{\prime}\right)$.

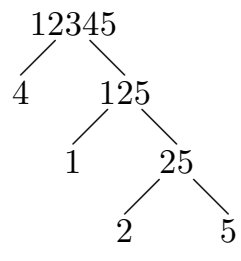

$T$

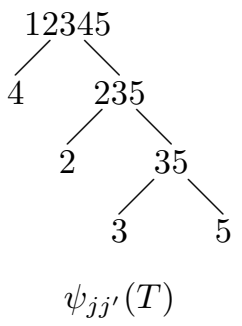

We now construct a bijection between binary total partitions and decomposition trees. Let $S \subset \mathbb{N}, j<\min (S)$, and $T \in \operatorname{BPP}(S, j)$. Define a tree $\phi(T)$ recursively as follows. If $|S|=1$, then $\phi(T)=T$. Otherwise, $\phi(T)$ is the tree

$$
\phi\left(\operatorname{Aug}_{j}\left(T^{(1)}\right)\right) \overbrace{\phi\left(\psi\left(\operatorname{Aug}_{m}\left(T^{(2)}\right)\right)\right)}^{\operatorname{rt}(T)}
$$

where $m=\max \left(\operatorname{rt}\left(T^{(1)}\right)\right)$. 
Theorem 7.3. For every $S \subset \mathbb{N}$ and $j<\min (S)$, the function $\phi$ is a bijection from $\operatorname{BPP}(S, j)$ to $\operatorname{Adm}(S \cup\{j\})$.

Proof. Let $T \in \operatorname{BPP}(S, j)$. Neither augmentation nor applying $\psi$ changes the shape of a tree, so by induction $\phi(T)$ has the same shape as $T$. Accordingly, if $X$ is a node of $T$, we may write $\phi(X)$ for the node of $\phi(T)$ in the same position as $X$.

First, we show that $\phi(T) \in \operatorname{Adm}(S \cup\{j\})$. If $|S|=1$, this is trivial. Otherwise, we make the inductive assumption that whenever $\left|S^{\prime}\right|<|S|$ and $j^{\prime}<\min \left(S^{\prime}\right)$ (that is, the members of $\operatorname{BPP}\left(S^{\prime}, j^{\prime}\right)$ are proper) we have $\phi\left(T^{\prime}\right) \in \operatorname{Adm}\left(S^{\prime} \cup\left\{j^{\prime}\right\}\right)$. In particular, the trees $\operatorname{Aug}_{j}\left(T^{(1)}\right)$ and $\psi\left(\operatorname{Aug}_{m}\left(T^{(2)}\right)\right)$ are proper, so by induction

$$
\begin{array}{lll}
\phi(T)^{(1)} & =\phi\left(\operatorname{Aug}_{j}\left(T^{(1)}\right)\right) & \in \operatorname{Adm}\left(X^{(1)} \cup\{j\}\right) \\
\phi(T)^{(2)} & =\phi\left(\psi\left(\operatorname{Aug}_{m}\left(T^{(2)}\right)\right)\right) & \in \operatorname{Adm}\left(X^{(2)} \cup\{m\}\right) .
\end{array}
$$

It is now routine to check that $X=S \cup\{j\}, X^{(1)}$ and $X^{(2)}$ satisfy the conditions of Proposition 6.3

Second, we show that $\phi$ is injective. If $|S|=1$ or 2 then $|\operatorname{BPP}(S, j)|=1$, so there is nothing to prove. Otherwise, we assume inductively that $\phi$ maps onto $\operatorname{BPP}\left(S^{\prime}, j\right)$ whenever $\left|S^{\prime}\right|<|S|$. Let $T, U \in \operatorname{BPP}(S, j)$ with $\phi(T)=\phi(U)$. Then

$$
\phi\left(\operatorname{Aug}_{j}\left(T^{(1)}\right)\right)=\phi(T)^{(1)}=\phi(U)^{(1)}=\phi\left(\operatorname{Aug}_{j}\left(U^{(1)}\right)\right) .
$$

By induction, we can cancel the $\phi$ 's from the outer terms, and $\mathrm{Aug}_{j}$ is a bijection, so $T^{(1)}=U^{(1)}$. Now, setting $m=\max \left(\operatorname{rt}\left(T^{(1)}\right)\right)=\max \left(\operatorname{rt}\left(U^{(1)}\right)\right)$, we have

$$
\phi\left(\psi\left(\operatorname{Aug}_{m}\left(T^{(1)}\right)\right)\right)=\phi(T)^{(2)}=\phi(U)^{(2)}=\phi\left(\psi\left(\operatorname{Aug}_{m}\left(U^{(1)}\right)\right)\right) .
$$

As before, it follows from the inductive hypothesis and the bijectivity of $\psi$ and $\operatorname{Aug}_{m}$ that $T^{(2)}=U^{(2)}$. Of course $\operatorname{rt}(T)=\operatorname{rt}(U)=S \cup\{j\}$, so $T=U$ as desired.

Finally, we show that $\phi$ is surjective. If $|S|=1$ or 2 , then $|\operatorname{Adm}(S \cup\{j\})|=1$, so there is nothing to prove. Otherwise, we make the inductive assumption that $\phi$ is surjective on $\operatorname{BPP}\left(S^{\prime}, j\right)$ whenever $\left|S^{\prime}\right|<|S|$. Suppose that $j<\min (S)$ and $U \in \operatorname{Adm}(S \cup\{j\})$. Note that $U^{(1)} \in \operatorname{Adm}\left(S^{(1)} \cup\{j\}\right)$ and $U^{(2)} \in \operatorname{Adm}\left(S^{(2)} \cup\{m\}\right)$, where $S^{(1)}$ and $S^{(2)}$ are nonempty disjoint sets with $S^{(1)} \cup S^{(2)}=S, m=\max \left(S^{(1)}\right)$, and $\max (S) \in S^{(2)}$. Let $k=\min \left(S^{(2)} \cup\{m\}\right)$ and

$$
\begin{array}{lll}
T^{(1)}=\operatorname{Aug}_{j}^{-1}\left(\phi^{-1}\left(U^{(1)}\right)\right) & \in \operatorname{BPP}\left(S^{(1)}\right), \\
T^{(2)}=\operatorname{Aug}_{m}^{-1}\left(\psi_{m k}^{-1}\left(\phi^{-1}\left(U^{(2)}\right)\right)\right) & \in \operatorname{BPP}\left(S^{(2)}\right) .
\end{array}
$$

Let $T$ be the tree

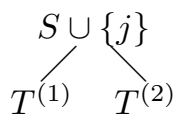

Then $\phi(T)=U$ and we are done.

For convenience, we summarize here our present knowledge about the simplicial complex $\Delta(n)$. Note that $\operatorname{dim} I_{n}=\operatorname{dim} J_{n}=2 n-3$ and $\operatorname{in}\left(I_{n}\right) \supset J_{n}$, so $\operatorname{deg} I_{n}=$ $\operatorname{deg} \operatorname{in}\left(I_{n}\right) \leq \operatorname{deg} J_{n}$. Therefore:

Theorem 7.4. The function $\theta=\phi \circ \mathrm{Aug}_{1}$ is a bijection from $\operatorname{BPP}([2, n])$ to $\operatorname{Adm}([1, n])$. In particular, $\Delta(n)$ has exactly

$$
\frac{(2 n-4) !}{2^{n-2}(n-2) !}
$$


facets. Moreover, this number equals the degree of the ideal $J_{n}$, and is an upper bound for the degree of $I_{n}$.

\section{A Shelling of $\Delta(n)$}

We prove in this section that the simplicial complex $\Delta(n)$ is shellable, hence Cohen-Macaulay, for all $n \geq 2$. We begin by recalling briefly the definition of shellability. (There are many equivalent definitions; see, e.g., [3 pp. 214-219].)

Definition 8.1. Let $\Delta$ be a pure simplicial complex. A total ordering $\succ$ of the facets of $\Delta$ is called a shelling if each facet $F$ has a subset $\operatorname{sh}(F)$ such that

(SH1) If $F \succ G$, then $\operatorname{sh}(F) \not \subset G$, and

(SH2) For each $e \in \operatorname{sh}(F)$, there is some $G \prec F$ such that $F \backslash G=\{e\}$.

We first fix some notation. Let $T$ be a decomposition tree. Denote by $\mathcal{L}(T)$ the set consisting of the root of $T$ together with all firstborn nodes. For $F$ a facet of $\Delta(n)$, we set $\mathcal{L}(F)=\mathcal{L}(\mathbf{T}(F))$.

Order the finite subsets of $\mathbb{N}$ as follows: $X>Y$ if $|X|>|Y|$, or if $|X|=|Y|$ and $\min (X \# Y) \in Y$. Let $F, G \in \Delta(n)$ be facets, with traversals

$$
\begin{aligned}
\operatorname{trav}(\mathbf{T}(F)) & =\left(X_{1}, \ldots, X_{N}\right), \\
\operatorname{trav}(\mathbf{T}(G)) & =\left(Y_{1}, \ldots, Y_{N}\right),
\end{aligned}
$$

where $N=2 n-3$. Let $k=\min \left\{i \mid X_{i} \neq Y_{i}\right\}$. Then we define a total order on facets by putting $F \succ G$ if $X_{k}>Y_{k}$. Note that in this case $X_{k}$ and $Y_{k}$ have the same pedigree.

We will prove that this ordering satisfies the conditions (SH1) and (SH2). First, we prove a fact to be used in the proof.

Lemma 8.2. Let $T$ be a decomposition tree on $V \subset \mathbb{N}$. Then the edge set $\mathbf{E}(\mathcal{L}(T))$ is a spanning tree of the complete graph on $V$.

Proof. Suppose that $\mathbf{E}(\mathcal{L}(T))$ contains a cycle $C$. Let $Y$ be the least common ancestor of the nodes in $T$ corresponding to edges in $C$, and let $e=\mathbf{E}(Y)$. Then the endpoints of $e$ are the minimum and maximum vertices of $C$, so $e$ lies on $C$ by Lemma 5.3 Let $e^{\prime}$ be the other edge of $C$ incident to the vertex $\max (V(C))=$ $\max (Y)$. The node $X$ corresponding to $e^{\prime}$ belongs to the right rib of $T \mid Y$, that is, the set

$$
\operatorname{rr}(T)=\left\{\operatorname{rt}(T), \operatorname{rt}(T)^{(2)}, \operatorname{rt}(T)^{(22)}, \ldots\right\} .
$$

In particular $X \notin \mathcal{L}(T)$, which is a contradiction. Therefore $\mathbf{E}(\mathcal{L}(T))$ does not contain any cycles. Since its cardinality is $n-1$, it is a spanning tree of $V$.

Theorem 8.3. The order $\succ$ is a shelling order on the facets of $\Delta(V)$, with $\operatorname{sh}(F)$ defined recursively by

$$
\operatorname{sh}(F)= \begin{cases}\emptyset & \text { if }|V| \leq 3 \\ \operatorname{sh}\left(F^{2}\right) & \text { if } V\left(F^{1}\right)=\left\{\min (V), \min _{2}(V)\right\} \\ \operatorname{sh}\left(F^{2}\right) \cup \mathcal{L}\left(F^{1}\right) & \text { if otherwise }\end{cases}
$$

where $\min _{2}(V)$ denotes the second smallest element of $V$. 
Proof. We may assume without loss of generality that $V=[1, n]$. In the first part of the proof, we will show that the order $\succ$ satisfies SH1. Let $F, G$ be facets of $\Delta(n)$ such that $F \succ G$. Put $T=\mathbf{T}(F), U=\mathbf{T}(G), \operatorname{trav}(T)=\left(X_{1}, \ldots, X_{N}\right)$, and $\operatorname{trav}(U)=\left(Y_{1}, \ldots, Y_{N}\right)$, with $X_{i}=Y_{i}$ for $i<k$ and $X_{k}>Y_{k}$. For ease of use, we abbreviate $X=X_{k}$ and $Y=Y_{k}$.

The parent nodes $X^{(P)}=Y^{(P)}$ have the same label, a set of cardinality $\geq 4$. If $X$ and $Y$ are the younger siblings of their parents, then $X^{(P 1)}=Y^{(P 1)}$ by definition of $k$, but then $X=Y$ by Remark 6.5 a contradiction. Therefore $X=X^{(P 1)}$ and $Y=Y^{(P 1)}$. In particular $E \subset \operatorname{sh}(F)$, where $E=\mathbf{E}(\mathcal{L}(T \mid X))$. Now $X>Y$, so in particular $X \neq\left\{\min \left(X^{(P)}\right), \min _{2}\left(X^{(P)}\right)\right\}$. Therefore $\mathbf{E}(X) \in E$.

We claim that $E \not \subset G$. It is sufficient to prove that $E \not \subset \mathbf{E}\left(U \mid Y^{(P)}\right)$, because if $Z \in U$ is not a descendant of $Y^{(P)}$, then the endpoints of $\mathbf{E}(Z)$ do not both belong to $Y^{(P)}=X^{(P)}$, so $\mathbf{E}(Z) \notin E$.

Suppose first that $\max (X)>\max (Y)$. Then $\max (X) \notin Z$ for all $Z \in U \mid Y$; $\min (X) \notin Z$ for all $Z \in U \mid Y^{(P 2)}$; and $\max (X)<\max \left(Y^{(P)}\right)$. Hence $\mathbf{E}(X) \notin$ $\mathbf{E}\left(U \mid Y^{(P)}\right)$, establishing the claim.

Now, suppose that $\max (X) \leq \max (Y)$. Let $v \in X \backslash Y$. By Lemma $8.2 E$ is a spanning tree of the vertices in $X$. In particular, $E$ contains a path $P$ from $v$ to $\min (X)=\min (Y)$. If $\max (X)=\max (Y)$, then $\max (Y)$ is a leaf of $E$ (since $\mathbf{E}(X)$ is the only edge having it as an endpoint), while if $\max (X)<\max (Y)$, then $\max (Y) \notin$ $V(E)$. In either case $\max (Y) \notin V(P)$. Note that $v \in Y^{(P 2)} \backslash Y$ and $\min (Y) \in$ $Y \backslash Y^{(P 2)}$. By Theorem 5.2 $\max (Y)$ is a cut-vertex of $\mathbf{E}\left(U \mid Y^{(P)}\right) \backslash\left\{\mathbf{E}\left(Y^{(P)}\right)\right\}$. So every path from $\min (Y)$ to $v$ in $\mathbf{E}\left(U \mid Y^{(P)}\right)$ must either pass through $\max (Y)$ or include the edge $\mathbf{E}\left(Y^{(P)}\right)$. But $P$ does neither of these things, so $P \not \subset \mathbf{E}\left(U \mid Y^{(P)}\right)$, establishing the claim and completing the proof that $\succ$ satisfies SH1.

For the second part of the proof, let $F \in \Delta(n)$ be a facet, $T=\mathbf{T}(F), e \in \operatorname{sh}(F)$, and $X$ the node of $T$ corresponding to $e$. We will show that $\Delta(n)$ contains a facet $G$ satisfying condition SH2. We proceed by induction on $n$; there is nothing to prove if $n \leq 3$.

Suppose first that $e \in \operatorname{sh}\left(F^{2}\right)$. By induction, the simplicial complex $\Delta\left(V\left(F^{2}\right)\right)$ has a facet $G^{2} \prec F^{2}$ such that $F^{2} \backslash G^{2}=\{e\}$. Therefore, the facet of $\Delta(n)$ given by $F^{1} \cup G^{2} \sqcup\{\{\min (V(F)), \max (V(F))\}\}$ satisfies SH2.

On the other hand, suppose that $V\left(F^{1}\right) \neq\{1,2\}$ and $e \in \mathcal{L}\left(F^{1}\right)$. We consider two separate cases: either $X$ has children or it does not.

Case 1: $X$ has children. Let $Y=X^{(P)}, E=\mathbf{E}(T \mid Y), F_{1}=\mathbf{E}\left(T \mid X^{(1)}\right)$, and $F_{2}=\mathbf{E}\left(T \mid X^{(2)}\right) \cup \mathbf{E}\left(T \mid Y^{(2)}\right)$. Note that $F_{1}$ is a facet of $\Delta\left(X^{(1)}\right)$, and $F_{2} \in$ $\Delta\left(X^{(2)} \cup Y^{(2)}\right)$. Moreover, since $X^{(2)} \cap Y^{(2)}=\left\{\max \left(X^{(2)}\right)\right\}$, we have

$$
\left|F_{2}\right|=\left(2\left|X^{(2)}\right|-3\right)+\left(2\left|Y^{(2)}\right|-3\right)=\operatorname{dim}\left(\Delta\left(X^{(2)} \cup Y^{(2)}\right)\right)
$$

so $\Delta\left(X^{(2)} \cup Y^{(2)}\right)$ has a facet $F_{2}^{\prime}$ of the form $F_{2} \cup\left\{e^{\prime}\right\}$. Let $G^{\prime}=F_{1} \cup F_{2}^{\prime} \cup\{\mathbf{E}(Y)\}$; this is a facet of $\Delta(Y)$ because

$$
X^{(1)} \cup\left(X^{(2)} \cup Y^{(2)}\right)=Y \quad \text { and } \quad X^{(1)} \cap\left(X^{(2)} \cup Y^{(2)}\right)=\left\{\max \left(X^{(1)}\right)\right\} .
$$

Let $G$ be the facet of $\Delta(V)$ obtained by replacing $T \mid Y$ with $\mathbf{T}\left(G^{\prime}\right)$. Then $F \backslash G=$ $\{e\}$. Moreover, the traversals of $\mathbf{T}(F)$ and $\mathbf{T}(G)$ first differ at $Y^{(1)}$, which is $X$ in $\mathbf{T}(F)$ and $X^{(1)}$ in $\mathbf{T}(G)$. Since $X^{(1)} \subsetneq X$, we have $F \succ G$, so $G$ satisfies SH2.

Case 2: $X$ is a leaf. In particular $|X|=2$. If $X \neq\left\{\min \left(X^{(P)}\right), \min _{2}\left(X^{(P)}\right)\right\}$, then replacing $X$ by that set produces a facet $G$ which is easily seen to satisfy SH2. 
Now suppose that $X=\left\{\min \left(X^{(P)}\right), \min _{2}\left(X^{(P)}\right)\right\}$. Put $X_{0}=X$ and $X_{i}=X_{i-1}^{(P)}$ for $i \geq 1$. Notice that $T^{(P)}$ is not in the right rib of $T$; if it were, it would have to be the root of $T$ (since $X$ is itself in the left subtree of $T$ ), which would imply that $X$ is the only node in the left subtree, hence not a member of $\operatorname{sh}(F)$. Accordingly, let $Y$ be the youngest ancestor of $X$ such that $X \in T \mid Y^{(1)}$; then $Y=X_{s}$ for some $s \geq 2$. (If no such $Y$ exists, then $X^{(P)} \in \operatorname{rr}(T)$, but $X \in \mathcal{L}(T)$ is in the left subtree of $\operatorname{rt}(T)$, so that $X^{(P)}=\operatorname{rt}(T)$.) Also, let $Z=X^{(P 2)}$. That is, $T \mid Y$ has the following form:

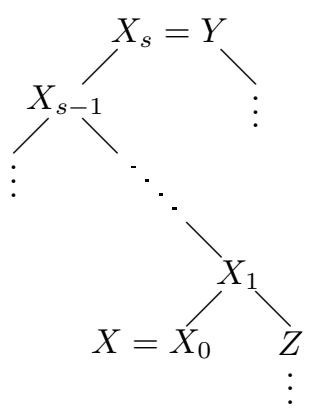

For $i \in[s-1]$, define $E_{i}=\mathbf{E}\left(T \mid X_{i}\right) \backslash\{e\}$. Each $\mathbf{E}\left(T \mid X_{i}\right)$ is a facet of $\Delta\left(X_{i}\right)$, hence 2 -connected by Theorem [5.2 so every $E_{i}$ is connected. On the other hand, we claim that $E_{i}$ is not 2 -connected. More specifically, we claim that for every $i$, the vertex $m=\max \left(X_{s-1}\right)$ is a cut-vertex separating $\min (X)$ and $\max (X)$. If $i=1$, the vertex $\min (X)$ is incident to exactly two edges in $\mathbf{E}\left(T \mid X_{1}\right)$, namely $\mathbf{E}(X)=e$ and $\mathbf{E}\left(X_{1}\right)=\left\{\min \left(X_{1}\right), \max \left(X_{1}\right)\right\}=\{\min (X), m\}$, so $m$ is the only neighbor of $\min (X)$ in $E_{1}$.

Now suppose that $1<i \leq s-1$. Note that both $\mathbf{E}\left(X_{1}\right)=\{\min (X), m\}$ and $\mathbf{E}(Z)=\{\max (X), \max (Z)=m\}$ belong to $E_{i}$. Suppose that $E_{i}$ contains a path $P$ from $\min (X)$ to $\max (X)$ which does not pass through $m$. Let $e^{\prime}$ be the edge incident to $\min (X)$ in $P$, and $W$ the corresponding node of $T$. Then $W$ is not one of the nodes $X_{j}$, since $m=\max \left(X_{j}\right)$ for all $j$. Also, $W \notin T \mid Z$, because $\min (X) \notin Z$. Therefore $W \in T \mid X_{j}^{(1)}$ for some $j \in[2, i]$. In particular $\min (X) \in X_{j}^{(1)} \cap X_{j-1}$, so $\min (X)=\max \left(X_{j}^{(1)}\right)$ and $\min (X) \neq \min (V(P))$. But then $P \cup\left\{\mathbf{E}\left(X_{1}\right), \mathbf{E}(Z)\right\}$ is a cycle in $E_{i}$ in which the smallest vertex, namely $\min (V(P))$, and the largest vertex, namely $m$, are not adjacent. This contradicts Lemma 5.3 and completes the proof of the claim.

Let $F_{1}$ be the block of $E_{s-1}$ containing the vertex $\min (Y)$, and let $F_{2}=\left(E_{s-1} \backslash\right.$ $\left.F_{1}\right) \cup \mathbf{E}\left(T \mid Y^{(2)}\right)$. Also let $V_{i}=V\left(F_{i}\right)$ for $i=1,2$. Note that

$$
\begin{aligned}
V_{1} \cup V_{2} & =Y, \\
V_{1} \cap V_{2} & =\{m\}, \quad \text { and } \\
V_{1} & \subsetneq X_{s-1} .
\end{aligned}
$$

For $i=1$ or 2 , let $F_{i}^{\prime}$ be a facet of $\Delta\left(V\left(F_{i}\right)\right)$ containing $F_{i}$. Now

$$
\left|F_{1}\right|+\left|F_{2}\right|=\left|F_{1} \cup F_{2}\right|=|\mathbf{E}(T \mid Y) \backslash\{\mathbf{E}(X), \mathbf{E}(Y)\}|=2|Y|-5
$$

and

$$
\left|F_{1}^{\prime}\right|+\left|F_{2}^{\prime}\right|=\left(2\left|V_{1}\right|-3\right)+\left(2\left|V_{2}\right|-3\right)=2|Y|-4,
$$


the last equality following from (17a) and (17b). Therefore $F_{1}^{\prime} \cup F_{2}^{\prime}=F_{1} \cup F_{2} \cup\left\{e^{\prime}\right\}$, for some edge $e^{\prime} \neq e$, and the face $G^{\prime}=F_{1}^{\prime} \cup F_{2}^{\prime} \cup\{\mathbf{E}(Y)\}$ is a facet of $\Delta(Y)$ with $\mathbf{E}(T \mid Y) \backslash G^{\prime}=\{e\}$. Let $G$ be the facet of $\Delta(V)$ obtained by replacing $T \mid Y$ with $\mathbf{T}\left(G^{\prime}\right)$. Then $F \backslash G=\{e\}$ as well. Furthermore, (17c) implies that $F \succ G$. Therefore $G$ is the desired facet satisfying SH2.

\section{The $h$-VECTOR of $\Delta(n)$}

Since $\Delta(n)$ is shellable, its $h$-vector

$$
h(\Delta(n))=(h(n, 0), h(n, 1), \ldots)
$$

has the following combinatorial interpretation [3 Corollary 5.1.14]: for any shelling order $\succ, h(n, k)$ is the number of facets $F \in \Delta(n)$ with $\left|\operatorname{sh}_{\succ}(F)\right|=k$. In this section, we prove that the numbers $h(n, k)$ have another, more elementary combinatorial interpretation: they enumerate perfect matchings by the number of long pairs. These numbers were first investigated by Kreweras and Poupard [8]; we discovered the connection using the On-Line Encyclopedia of Integer Sequences [11. We begin by obtaining a recurrence for $h(n, k)$, using the description of facets and the shelling order of Theorem 8.3. We then show that the recurrence is equivalent to one enumerating matchings by the number of long pairs

Let $F \in \Delta(n)$ be a facet and $T=\mathbf{T}(F)$ the corresponding decomposition tree. Define $\operatorname{sh}(T)=\{X \in T \mid \mathbf{E}(X) \in \operatorname{sh}(F)\}$.

Let $\theta: \operatorname{BPP}[2, n] \rightarrow \operatorname{Adm}([1, n])$ be the bijection of Theorem 7.4 Recall that $T$ and $\theta(T)$ have the same shape, so we may define $\operatorname{sh}(U)$ to be the set of nodes in the same positions as the nodes in $\operatorname{sh}\left(\theta^{-1}(U)\right)$.

Recall the definition (16) of the right rib $\operatorname{rr}(T)$ of a binary tree. As before, denote the second smallest element of a set $S$ by $\min _{2}(S)$.

Lemma 9.1. Let $F \in \Delta(n)$ be a facet, $T=\mathbf{T}(F)$ the corresponding decomposition tree, and $X$ a firstborn node of $T$. Then:

(i) $X \notin \operatorname{sh}(T)$ if and only if $X=\left\{\min \left(X^{(P)}\right), \min _{2}\left(X^{(P)}\right)\right\}$ and $X^{(P)} \in \operatorname{rr}(T)$.

(ii) $\theta(X) \notin \operatorname{sh}(\theta(T))$ if and only if $\theta(X)=\left\{\min \left(\theta(X)^{(P)}\right)\right\}$ and $\theta(X)^{(P)} \in$ $\operatorname{rr}(\theta(T))$.

Proof. (i) The first condition implies that $X \notin \operatorname{sh}\left(X^{(P)}\right)$; the second condition implies that $\operatorname{sh}\left(X^{(P)}\right)=\operatorname{sh}(F) \cap \mathbf{E}\left(T \mid X^{(P)}\right)$. Together, they imply that $X \notin \operatorname{sh}(F)$. On the other hand, suppose that either or both conditions fail. Let $Y$ be the youngest ancestor of $X$ which does not belong to $\operatorname{rr}(T)$. Then $Y$ is firstborn, and $X \in \operatorname{sh}\left(T \mid Y^{(P)}\right) \subset \operatorname{sh}(F)$.

(ii) This is obtained by translating the conditions from Lemma 9.1 (i) into conditions on $\theta(T)$, using the definition of $\theta$.

Let $T \in \operatorname{BPP}[2, n-1]$ and $X \in T$. Define a tree $\gamma(T, X) \in \operatorname{BPP}[1, n-1]$ as follows: replace $T \mid X$ with the tree

$$
\overbrace{1}^{X \cup\{1\}}
$$

and append 1 to the label of every ancestor of $X$. The map

$$
\gamma:\{(T, X) \mid T \in \operatorname{BPP}[2, n-1], X \in T\} \rightarrow \operatorname{BPP}[1, n-1]
$$


is clearly one-to-one, and these sets have the same cardinality, so $\gamma$ is a bijection. By incrementing all numbers in the labels of all nodes of $\gamma(T, X)$, we obtain a bijection $\tilde{\gamma}$ which maps pairs $(T, X)$ as above to $\mathrm{BPP}[2, n]$. Moreover,

$$
\operatorname{sh}(\tilde{\gamma}(T, X))= \begin{cases}\operatorname{sh}(T) & \text { if } X=\operatorname{rt}(T), \\ \operatorname{sh}(T) \cup\{1, X\} & \text { if } X \text { is firstborn and } X \notin \operatorname{sh}(T), \\ \operatorname{sh}(T) \cup\{1\} & \text { otherwise, }\end{cases}
$$

where $\operatorname{sh}(T)=\operatorname{sh}(\theta(T))$ for a binary total partition $T$. Accordingly, we may formulate a recurrence for the numbers $h(n, k)$. Suppose that $U=\tilde{\gamma}(T, X) \in \operatorname{BPP}[2, n]$ (so $T \in \mathrm{BPP}[2, n-1]$ has $2 n-5$ nodes, of which $n-3$ are firstborn) and $|\operatorname{sh}(U)|=$ $k$. Then one of the following is true: either

- $|\operatorname{sh}(T)|=k$ and $X=\operatorname{rt}(T)$;

- $|\operatorname{sh}(T)|=k-1$ and $X$ is either one of the $n-3$ secondborn nodes of $T$, or one of the $k-1$ members of $\operatorname{sh}(T)$; or

- $|\operatorname{sh}(T)|=k-2$ and $X$ is one of the $(n-3)-(k-2)$ firstborn nodes of $T$ which do not belong to $\operatorname{sh}(T)$.

Therefore $h(n, k)$ is defined by the recurrence

$$
h(n, k)=h(n-1, k)+(n+k-4) h(n-1, k-1)+(n-k-1) h(n-1, k-2)
$$

with base cases $h(n, k)=0$ if $k<0$ or $k>n-2, h(n, 0)=1$ for $n \geq 2$.

Let $n \in \mathbb{N}$. A matching on $[1,2 n]$ is a partition of $[1,2 n]$ into $n$ pairs. A pair $\{i, j\}$ is short if $|i-j|=1$; otherwise it is long. Define

$$
\begin{aligned}
M(n) & =\{\text { matchings on }[1,2 n]\} \\
M(n, k) & =\{X \in M(n) \mid X \text { has } k \text { long pairs }\} \\
m(n, k) & =|M(n, k)| .
\end{aligned}
$$

Kreweras and Poupard [8] gave recurrences and a closed formula for $m(n, k)$. We will use a slightly different argument to show that $m(n, k)$ is given by a recurrence equivalent to (19).

Note first that $m(n, 0)=1$ for all $n$, because the only matching on $[1,2 n]$ with no long pairs is $\{\{1,2\},\{3,4\}, \ldots,\{2 n-1,2 n\}\}$.

Let $X \in M(n-1)$ and $2 \leq p \leq 2 n$. We define a matching $X^{p} \in M(n)$ by inserting the pair $\{1, p\}$ into $X$ as follows. First, we relabel $X$ to obtain a matching on $[1,2 n] \backslash\{1, p\}$; this amounts to replacing each $x \in[1,2 n-2]$ by $x+1$ if $x<p-1$, or by $x+2$ if $x \geq p-1$. Having done this, we obtain $X^{p}$ by adjoining the pair $\{1, p\}$.

The map sending $(X, p)$ to $X^{p}$ is a bijection from $M(n-1) \times[2,2 n] \rightarrow M(n)$, so by induction $|M(n)|=(2 n) ! / 2^{n} n$ !. Moreover, we can derive a recurrence for $m(n, k)$. Let $X \in M(n, k)$. If $p=2$, then $X^{p} \in M(n+1, k)$. If $\{p-2, p-1\} \in X$ (which occurs for $n-k$ values of $p$ ), then $X^{p} \in M(n+1, k+2)$ (because $\{p-2, p-1\}$ becomes the long pair $\{p-1, p+1\}$ in $\left.X^{p}\right)$. For the other $n+k$ values of $p$, we have $X^{p} \in M(n+1, k+1)$. Accordingly, $m(n, k)$ is defined by the recurrence

$$
m(n, k)=m(n-1, k)+(n+k-2) m(n-1, k-1)+(n-k+1) m(n-1, k-2)
$$

with base cases $m(n, k)=0$ if $k<0$ or $k>n$, and $m(n, 0)=1$ for all $n \geq 0$.

Theorem 9.2. Let $n \geq 2$ and $0 \leq k \leq n-2$. Then $h(n, k)=m(n-2, k)$, the number of matchings on $[1,2 n-4]$ with $k$ long pairs. 
Proof. When $k=0$ or $n=2$, equality is immediate from the base cases. Otherwise, assume that $h\left(n^{\prime}, k\right)=m\left(n^{\prime}-2, k\right)$ for $n^{\prime}<n$. Then (19) and (20) give

$$
\begin{aligned}
m(n & -2, k) \\
& =m(n-3, k)+(n+k-4) m(n-3, k-1)+(n-k-1) m(n-3, k-2) \\
& =h(n-1, k)+(n+k-4) h(n-1, k-1)+(n-k-1) h(n-1, k-2) \\
& =h(n, k) .
\end{aligned}
$$

\section{A Recursive Lower Bound for Degree}

In this section, we return to geometry. To complete the proof of the main theorem, we must show that $J_{n}$ is the initial ideal of an ideal defining the slope variety $\tilde{\mathcal{S}}\left(K_{n}\right)$ scheme-theoretically. The key ingredient is to bound the (geometric) degree of $\tilde{\mathcal{S}}\left(K_{n}\right)$ from below. We do this by studying a family of flattened slope varieties $\tilde{\mathcal{S}}(n, k)$ forming a filtration of $\tilde{\mathcal{S}}\left(K_{n}\right)$ : that is,

$$
\tilde{\mathcal{S}}\left(K_{n}\right)=\tilde{\mathcal{S}}(n, 1) \supset \tilde{\mathcal{S}}(n, 2) \supset \cdots \supset \tilde{\mathcal{S}}(n, n) .
$$

We obtain a recursive lower bound $e(n, k) \leq \operatorname{deg} \tilde{\mathcal{S}}(n, k)$. In Section 11] we interpret the numbers $e(n, k)$ combinatorially and show that they actually give the degree exactly.

We begin by recalling some standard facts about the geometric notion of degree. Let $X \subset \mathbb{A}^{N}$ (or $\mathbb{P}^{N}$ ) be an algebraic set of dimension $d$. The degree of $X$, denoted $\operatorname{deg} X$, is the number of intersection points of $X$ with a generic affine linear subspace of codimension $d$. We extend this definition to locally closed sets $X, Y$ by putting $\operatorname{deg} X=\operatorname{deg} \bar{X}$. Degree is multiplicative on products: $\operatorname{deg}(X \times Y)=$ $(\operatorname{deg} X)(\operatorname{deg} Y)$. Moreover, if $H$ is a hyperplane, then $\operatorname{deg}(X \cap H) \leq \operatorname{deg} X$. Finally, if $X$ is the union of locally closed sets $C_{1}, \ldots, C_{k}$ (for instance, if these are the irreducible components of $X$ ), then the degree of $X$ is the sum of the degrees of all those $C_{i}$ whose dimension equals $\operatorname{dim} X$.

We will also need some general facts about graph varieties; for details, see [10]. An (affine) picture $\mathbf{P}$ of $K_{n}$ consists of $n$ points $\mathbf{P}(1), \ldots, \mathbf{P}(n)$ in the plane $\mathbb{A}^{2}$, and $\left(\begin{array}{l}n \\ 2\end{array}\right)$ non-vertical lines $\mathbf{P}(1,2), \ldots, \mathbf{P}(n-1, n)$, subject to the conditions $\mathbf{P}(i), \mathbf{P}(j) \in \mathbf{P}(i j)$ for all $i, j$. The set of all pictures is the affine picture variety $\tilde{\mathcal{V}}\left(K_{n}\right)$. The affine slope variety $\tilde{\mathcal{S}}\left(K_{n}\right)$ is thus obtained by projecting $\tilde{\mathcal{V}}\left(K_{n}\right)$ on affine coordinates corresponding to the slopes of the lines $\mathbf{P}(i j)$. We write $\phi$ for the surjection $\tilde{\mathcal{V}}\left(K_{n}\right) \rightarrow \tilde{\mathcal{S}}\left(K_{n}\right)$, and refer to a point in $\tilde{\mathcal{S}}\left(K_{n}\right)$ as a slope picture $\mathbf{m}=\left(m_{i j}\right)$.

A partition of a set $B$ is a collection of sets $\mathcal{A}=\left\{A_{i}\right\}$ such that $B$ is the disjoint union of the $A_{i}$. For each partition $\mathcal{A}$ of $[1, n]$, there is a corresponding cellule $\tilde{\mathcal{V}}_{\mathcal{A}}\left(K_{n}\right)$ defined by

$$
\tilde{\mathcal{V}}_{\mathcal{A}}\left(K_{n}\right)=\left\{\begin{array}{l|l}
\mathbf{P} \in \tilde{\mathcal{V}}\left(K_{n}\right) & \begin{array}{l}
\mathbf{P}(i)=\mathbf{P}(j) \text { if and only if } \\
i, j \text { belong to the same part of } \mathcal{A}
\end{array}
\end{array}\right\} .
$$

Thus $\tilde{\mathcal{V}}\left(K_{n}\right)$ is the disjoint union of the cellules $\tilde{\mathcal{V}}_{\mathcal{A}}\left(K_{n}\right)$ as $\mathcal{A}$ ranges over all partitions. Of particular importance are the discrete cellule, corresponding to the partition of $[1, n]$ into $n$ singleton sets, and the indiscrete cellule, corresponding to the partition with only one part. 
Lemma 10.1. Let $\mathbf{m} \in \tilde{\mathcal{S}}\left(K_{n}\right)$. Then there exists a picture $\mathbf{P} \in \phi^{-1}(\mathbf{m})$ in which not all points $\mathbf{P}(1), \ldots, \mathbf{P}(n)$ are the same. Equivalently, $\phi$ remains surjective if the indiscrete cellule is deleted from its domain.

Proof. The equations defining a picture of $G$ may be written in matrix form as $M X=0$, where $M$ is a matrix whose entries are linear forms in the variables $m_{e}$ (as in (4) ) and $X$ is the column vector $\left[x_{i}-x_{1}\right]_{2 \leq i \leq n}$. Every maximal minor of $M$ corresponds to a subset of $E\left(K_{n}\right)$ of cardinality $2 n-2$, which must contain at least one rigidity circuit. If $\mathbf{m} \in \tilde{\mathcal{S}}(G)$, then every maximal minor of $M(\mathbf{m})$ vanishes. Therefore $M(\mathbf{m})$ has a nonzero nullvector $X$, which gives the $x$-coordinates (up to translation) of a picture $\mathbf{P}$ not in the indiscrete cellule.

For $1 \leq k \leq n$, define an algebraic subset of $\tilde{\mathcal{S}}\left(K_{n}\right)$ by

$$
\tilde{\mathcal{S}}(n, k)=\left\{\mathbf{m} \in \tilde{\mathcal{S}}\left(K_{n}\right) \mid m_{i j}=0 \text { for } 1 \leq i<j \leq k\right\} .
$$

Note that $\tilde{\mathcal{S}}(n, 1)=\tilde{\mathcal{S}}\left(K_{n}\right)$.

Proposition 10.2. Let $n \geq 2$ and $1 \leq k \leq n$. Then

$$
\operatorname{dim} \tilde{\mathcal{S}}(n, k)= \begin{cases}0 & \text { if } n=k, \\ 2 n-k-2 & \text { if } n>k\end{cases}
$$

Proof. If $n=k$, then $\tilde{\mathcal{S}}(n, k)$ is a point. If $k=1$, then $\operatorname{dim} \tilde{\mathcal{S}}(n, k)=\operatorname{dim} \tilde{\mathcal{S}}\left(K_{n}\right)=$ $2 n-3=2 n-k-2$. These two observations complete the proof for $n=2$. For $n>2$, we assume inductively that the formula holds for all smaller $n$ and all $k$. For each partition $\mathcal{A}$ of $[1, n]$, define a closed subset $F_{\mathcal{A}}$ of $\tilde{\mathcal{V}}_{\mathcal{A}}\left(K_{n}\right)$ by

$$
F_{\mathcal{A}}=\phi^{-1}(\tilde{\mathcal{S}}(n, k)) \cap \tilde{\mathcal{V}}_{\mathcal{A}}\left(K_{n}\right) .
$$

Note that Lemma 10.1 implies that $\tilde{\mathcal{S}}(n, k)=\bigcup_{\mathcal{A}} \phi\left(F_{\mathcal{A}}\right)$, where $\mathcal{A}$ ranges over all partitions with at least two parts. In general, we may write $\mathcal{A}$ in the form

$$
\mathcal{A}=\left\{A_{1} \sqcup B_{1}, \ldots, A_{s} \sqcup B_{s}\right\}
$$

where the $A_{i}\left(\right.$ resp. $\left.B_{i}\right)$ form a partition of $[1, k]($ resp. $[k+1, n])$, with some $A_{i}$ (resp. $B_{i}$ ) allowed to be empty. Let $a_{i}=\left|A_{i}\right|$ and $b_{i}=\left|B_{i}\right|$. We may order the parts of $\mathcal{A}$ in such a way that

$$
\begin{array}{llll}
a_{i}>0, & b_{i}>0 & \text { for } & i \in[1, p] \\
a_{i}>0, & b_{i}=0 & \text { for } & i \in[p+1, q] \\
a_{i}=0, & b_{i}=1 & \text { for } & i \in[q+1, r] \\
a_{i}=0, & b_{i}>1 & \text { for } & i \in[r+1, s]
\end{array}
$$

for some $p \leq q \leq r \leq s$. Note also that $q \leq k$. A picture $\mathbf{P} \in F_{\mathcal{A}}$ is therefore given by $s$ distinct points in $\mathbb{A}^{2}$, of which $q$ lie on a common horizontal line, together with the slopes of the lines $\mathbf{P}(e)$ such that the endpoints of the edge $e$ are in the same part of $\mathcal{A}$ and are not both $\leq k$. This amounts to specifying $s x$-coordinates and $s-q+1 y$-coordinates for the points $\mathbf{P}(i)$; a point in $\tilde{\mathcal{S}}\left(a_{i}+b_{i}, a_{i}\right)$ for each $i \in[1, p]$; and a point in $\tilde{\mathcal{S}}\left(K_{b_{i}}\right)$ for each $i \in[r+1, s]$. By the inductive hypothesis, 
we have

$$
\begin{aligned}
\operatorname{dim} F_{\mathcal{A}} & =(2 s-q+1)+\sum_{i=1}^{p}\left(2 b_{i}-a_{i}-2\right)+\sum_{i=r+1}^{s}\left(2 b_{i}-3\right) \\
& =-2 p-s-q+3 r+1+2\left(\sum_{i=1}^{p} b_{i}+\sum_{i=r+1}^{s} b_{i}\right)-\sum_{i=1}^{p} a_{i} \\
& =3 r-2 p-q-s+1+2(n-k-(r-q))-\sum_{i=1}^{p} a_{i} \\
& =2 n-2 k-2 p+q+r-s+1-\sum_{i=1}^{p} a_{i} \\
\leq & 2 n-2 k-2 p+q+r-s+1 \\
& =(2 n-k+1)+(-k-2 p+q+r-s) \\
\leq & 2 n-k+1
\end{aligned}
$$

because $r \leq s$ and $q \leq k$. Every fiber of $\phi$ has dimension at least 3 , so $\operatorname{dim} \phi\left(F_{\mathcal{A}}\right) \leq$ $2 n-k-2$. Since $\tilde{\mathcal{S}}(n, k)$ is the disjoint union of the $F_{\mathcal{A}}$, its dimension is at most $2 n-k-2$ as well. On the other hand, if $\mathcal{A}$ is the discrete partition into $n$ singleton sets, then $p=0, q=k$, and $r=s=n$. So equality holds throughout the preceding calculation, and $\operatorname{dim} \tilde{\mathcal{S}}(n, k)=2 n-k-2$.

Define

$$
\tilde{\mathcal{S}}^{\prime}(n, k)=\left\{\mathbf{m} \in \tilde{\mathcal{S}}(n, k) \mid m_{1, k+1}=0\right\} .
$$

This set is the intersection of $\tilde{\mathcal{S}}(n, k)$ with a hyperplane, so its degree is a lower bound for that of $\tilde{\mathcal{S}}(n, k)$. We will calculate the degree of $\tilde{\mathcal{S}}^{\prime}(n, k)$ by identifying its irreducible components of maximal dimension. First, we calculate the dimension of $\tilde{\mathcal{S}}^{\prime}(n, k)$.

Proposition 10.3. Suppose $n>k \geq 1$ and $n \geq 2$. Then $\operatorname{dim} \tilde{\mathcal{S}}^{\prime}(n, k)=2 n-k-3$.

Proof. Intersecting $\tilde{\mathcal{S}}(n, k)$ with a codimension-1 hyperplane can lower the dimension by at most 1 . Hence for all $n$ and $k$, we have $\operatorname{dim} \tilde{\mathcal{S}}^{\prime}(n, k) \geq 2 n-k-3$.

For the reverse inequality, we induct on $n$. If $n=2$ and $k=1$, then $2 n-k-3=0$, and indeed $\tilde{\mathcal{S}}^{\prime}(2,1)=\tilde{\mathcal{S}}(2,2)$ is a point. Now suppose that $n>2$ and that the formula holds for all smaller $n$ and all $k$. For each partition $\mathcal{A}$ of $[1, n]$, define a closed subset $F_{\mathcal{A}}^{\prime}$ of $\tilde{\mathcal{V}}_{\mathcal{A}}\left(K_{n}\right)$ by

$$
F_{\mathcal{A}}^{\prime}=\phi^{-1}\left(\tilde{\mathcal{S}}^{\prime}(n, k)\right) \cap \tilde{\mathcal{V}}_{\mathcal{A}}\left(K_{n}\right) .
$$

By Lemma 10.1 $\tilde{\mathcal{S}}^{\prime}(n, k)=\bigcup_{\mathcal{A}} \phi\left(F_{\mathcal{A}}^{\prime}\right)$, where $\mathcal{A}$ ranges over all partitions of $n$ with at least two parts. We need to show that $\operatorname{dim} F_{\mathcal{A}}^{\prime} \leq 2 n-k$ for all such $\mathcal{A}$. In general, we may write

$$
\mathcal{A}=\left\{\{k+1\} \sqcup A_{1} \sqcup B_{1}, A_{2} \sqcup B_{2}, \ldots, A_{s} \sqcup B_{s}\right\}
$$

where the $A_{i}\left(\right.$ resp. $\left.B_{i}\right)$ form a partition of $[1, k]($ resp. $[k+2, n])$, with some $A_{i}$ (resp. $B_{i}$ ) allowed to be empty. Let $a_{i}=\left|A_{i}\right|$ and $b_{i}=\left|B_{i}\right|$. Order the parts of $\mathcal{A}$ 
so that for some $p \leq q \leq r \leq s$,

$$
\begin{array}{llll}
a_{i}>0, & b_{i}>0 & \text { for } & i \in[2, p] ; \\
a_{i}>0, & b_{i}=0 & \text { for } & i \in[p+1, q] ; \\
a_{i}=0, & b_{i}=1 & \text { for } & i \in[q+1, r] ; \\
a_{i}=0, & b_{i}>1 & \text { for } & i \in[r+1, s] .
\end{array}
$$

If $1 \in A_{1}$, then a picture $\mathbf{P} \in F_{\mathcal{A}}^{\prime}$ is given by the following data: $s$ distinct points in $\mathbb{A}^{2}$, of which $q$ lie on a common horizontal line (so there are $s-q+1 x$-coordinates and $s y$-coordinates); a point in $\tilde{\mathcal{S}}^{\prime}\left(1+a_{1}+b_{1}, a_{1}\right)$; a point in $\tilde{\mathcal{S}}\left(a_{i}+b_{i}, a_{i}\right)$ for each $i \in[2, p]$; and a point in $\tilde{\mathcal{S}}\left(K_{b_{i}}\right)$ for each $i \in[r+1, s]$. By induction,

$$
\operatorname{dim} F_{\mathcal{A}}^{\prime}=(2 s-q+1)+\left(a_{1}+2 b_{1}-1\right)+\sum_{i=2}^{p}\left(a_{i}+2 b_{i}-2\right)+\sum_{i=r+1}^{s}\left(2 b_{i}-3\right) .
$$

Now a simple calculation, which we omit because of its similarity to that of Proposition 10.2 shows that $\operatorname{dim} F_{\mathcal{A}}^{\prime} \leq 2 n-k$ as desired.

If $1 \notin A_{1}$, then a picture $\mathbf{P} \in F_{\mathcal{A}}^{\prime}$ is given by the following data: $s$ distinct points in $\mathbb{A}^{2}$, of which $q$ lie on a common horizontal line; a point in $\tilde{\mathcal{S}}\left(a_{i}+b_{i}, a_{i}\right)$ for each $i \in[1, p]$; and a point in $\tilde{\mathcal{S}}\left(K_{b_{i}}\right)$ for each $i \in[r+1, s]$. Therefore

$$
\begin{aligned}
\operatorname{dim} F_{\mathcal{A}}^{\prime} & =(2 s-q+1)+\sum_{i=1}^{p}\left(a_{i}+2 b_{i}-2\right)+\sum_{i=r+1}^{s}\left(2 b_{i}-3\right) \\
& =(-2 p-q+3 r-s+1)+\sum_{i=1}^{p} a_{i}+2\left(\sum_{i=1}^{p} b_{i}+\sum_{i=r+1}^{s} b_{i}\right) \\
& =2 n-2 k-2 p+q+r-s-1+\sum_{i=1}^{p} a_{i} .
\end{aligned}
$$

Note that $\sum_{i=1}^{p} a_{i} \leq k-(q-p)$, since $\left\{A_{1}, \ldots, A_{q}\right\}$ is a partition of $[1, k]$ with no empty parts. Therefore $\operatorname{dim} F_{\mathcal{A}}^{\prime} \leq 2 n-k-p+r-s-1<2 n-k$ as desired.

Theorem 10.4. Let $n \geq 2$ and $k \in[1, n]$. Then

$$
\operatorname{deg} \tilde{\mathcal{S}}(n, k) \geq e(n, k),
$$

where $e(n, n)=1$ for all $n ; e(2,1)=1$; and

$$
\begin{aligned}
e(n, k) & =e(n, k+1)+e(n-1, k-1) \\
& +\sum_{t=1}^{k-1} \sum_{u=0}^{n-k-2}\left(\begin{array}{c}
k-1 \\
t
\end{array}\right)\left(\begin{array}{c}
n-k-1 \\
u
\end{array}\right) e(t+u+1, t) e(n-t-u, k-t+1) .
\end{aligned}
$$

Proof. For all $n$, the variety $\tilde{\mathcal{S}}(n, n)$ is a point, so it has degree $1=e(n, n)$. The variety $\tilde{\mathcal{S}}(2,1)=\tilde{\mathcal{S}}\left(K_{2}\right)$ is a line, whose degree is again $1=e(2,1)$. Now suppose that $n>k$ and $n>2$. As noted before, $\operatorname{deg} \tilde{\mathcal{S}}^{\prime}(n, k) \leq \operatorname{deg} \tilde{\mathcal{S}}(n, k)$. The idea of the proof is to write $\tilde{\mathcal{S}}^{\prime}(n, k)$ as a finite union of locally closed subsets. Once we have done this, we may obtain a lower bound for $\operatorname{deg} \tilde{\mathcal{S}}^{\prime}(n, k)$ by summing lower bounds for the degrees of those locally closed subsets of dimension $2 n-k-3$.

First, note that $\tilde{\mathcal{S}}^{\prime}(n, k) \supset \tilde{\mathcal{S}}(n, k+1)$, and that this second set has dimension $2 n-k-3$. 
Second, let $\mathbf{m}=\left(m_{i j}\right)$ be a slope picture in $\tilde{\mathcal{S}}^{\prime}(n, k) \backslash \tilde{\mathcal{S}}(n, k+1)$. That is, $m_{i j}=0$ for $1 \leq i<j \leq k$, and $m_{1, k+1}=0$, but $m_{2, k+1}, \ldots, m_{k, k+1}$ are not all zero. Define two sets of vertices $T(\mathbf{m}), U(\mathbf{m})$ by

$$
\begin{aligned}
& T(\mathbf{m})=\left\{i \in[2, k] \mid m_{i, k+1} \neq 0\right\}, \\
& U(\mathbf{m})=\left\{i \in[k+2, n] \mid \mathbf{P}(i)=\mathbf{P}(k+1) \text { for all } \mathbf{P} \in \phi^{-1}(\mathbf{m})\right\} .
\end{aligned}
$$

Then $T(\mathbf{m}) \neq \emptyset$, and $\tilde{\mathcal{S}}^{\prime}(n, k) \backslash \tilde{\mathcal{S}}(n, k+1)$ is the disjoint union of the sets

$$
S_{T, U}=\left\{\mathbf{m} \in \tilde{\mathcal{S}}^{\prime}(n, k) \backslash \tilde{\mathcal{S}}(n, k+1) \mid T(\mathbf{m})=T, U(\mathbf{m})=U\right\}
$$

for $\emptyset \neq T \subset[2, k]$ and $U \subset[k+2, n]$. On $\phi^{-1}\left(S_{T, U}\right)$, one has $\mathbf{P}(i)=\mathbf{P}(k+1)$ for all $i \in T \cup U$. Thus the data for a picture in $\phi^{-1}\left(S_{T, U}\right)$ consists of $n-|T|-|U|$ points, of which $k-|T|+1$ must lie on a common horizontal line, together with the slopes of lines corresponding to edges with both endpoints in $T \cup U$. Applying the surjective map $\phi$, we see that $S_{T, U}$ has the form

$$
S_{T, U} \cong \tilde{\mathcal{S}}(n-t-u, k-t+1) \times \tilde{\mathcal{S}}(t+u+1, t)
$$

where $t=|T|$ and $u=|U|$. If $U \neq[k+2, n]$, then $n-t-u>k-t+1$. Applying Proposition 10.2 to (22), one obtains

$$
\begin{aligned}
\operatorname{dim} S_{T, U} & =(2(n-t-u)-(k-t+1)-2)+(2(t+u+1)-t-2) \\
& =2 n-k-3 .
\end{aligned}
$$

If $U=[k+2, n]$, then $n-t-u=k-t+1$. Now the first factor in (22) is a single point, so applying Proposition 10.2 yields

$$
\operatorname{dim} S_{T, U}=2(t+u+1)-t-2=(2 n-k-3)+(t-k+1) .
$$

This is strictly less than $2 n-k-3$ unless $T=[2, k]$, in which case $S_{T, U} \cong \tilde{\mathcal{S}}(n-$ $1, k-1)$. Putting all this together, we obtain

$$
\begin{aligned}
\operatorname{deg} \tilde{\mathcal{S}}^{\prime}(n, k) \geq \operatorname{deg} \tilde{\mathcal{S}}(n, k+1)+\operatorname{deg} \tilde{\mathcal{S}}(n-1, k-1) \\
+\sum_{\substack{\emptyset \neq T \subset[2, k] \\
U \subsetneq[k+2, n]}} \operatorname{deg} \tilde{\mathcal{S}}(n-t-u, k-t+1) \operatorname{deg} \tilde{\mathcal{S}}(t+u+1, t) .
\end{aligned}
$$

Now, summing over $t$ and $u$ instead of $T$ and $U$, and multiplying the summand by the appropriate binomial coefficients to reflect the number of ways of choosing the sets $T$ and $U$, one obtains the desired recurrence.

\section{Decreasing Planar Trees}

The recurrence established in Theorem 10.4 has a combinatorial interpretation in terms of decreasing planar trees. We begin with some general facts about these objects, recalling the general definitions and terminology for rooted planar trees given in Section [6]

Definition 11.1. Let $V$ be a finite subset of $\mathbb{N}$. A decreasing planar tree on $V$ is a rooted planar tree $T$ with the following property: if $v, w$ are nodes of $T$ with $v$ an ancestor of $w$, then $v>w$. (In particular, $T$ is rooted at the maximum element of $V$.) The set of all decreasing planar trees on $V$ is denoted $\operatorname{DPT}(V)$; as usual we abbreviate $\operatorname{DPT}([1, n])$ by $\operatorname{DPT}(n)$. 
The number of decreasing planar trees is given, once again, by the double factorial numbers:

$$
|\operatorname{DPT}(n)|=(2 n-3)(2 n-5) \cdots(3)(1)=\frac{(2 n-2) !}{(n-1) ! 2^{n-1}}
$$

(This is an elementary combinatorial exercise; see, e.g., [12 pp. 13-16].) We will obtain a recurrence enumerating decreasing planar trees on $V$ by the largest leaf statistic: $L(T)=\max \{i \in V \mid i$ is a leaf $\}$. Let

$$
\begin{aligned}
\operatorname{DPT}(n, k) & =\{T \in \mathrm{DPT}(n) \mid L(T) \leq k\} \\
d(n, k) & =|\operatorname{DPT}(n, k)| .
\end{aligned}
$$

Note that $d(n, 1)=1$ for all $n$, since if $L(T)=1$ then the tree $T$ can only be the path in which each $j>1$ has the unique child $j-1$. Moreover, if $n \geq 2$, then $\operatorname{DPT}(n, n)=\operatorname{DPT}(n, n-1)=\operatorname{DPT}(n)$. In addition, $d(n, k)=0$ if $n>0$ and $k \notin[1, n]$. Conventionally, we put $d(0, k)$ for all $k$.

If $T_{1}$ and $T_{2}$ are rooted planar trees and $X$ is a node of $T_{2}$, we may graft $T_{1}$ to $T_{2}$ by attaching it as the oldest subtree of $X$. We denote the resulting tree by $T_{1} \nearrow_{X} T_{2}$, suppressing the subscript if $X=\operatorname{rt}\left(T_{2}\right)$ (the most common case). To illustrate this operation, let $T$ be the binary tree pictured in Example 6.2 Let $T_{1}$ be the subtree rooted at the node labeled 245, and let $T_{2}$ be the tree obtained from $T$ by deleting $T_{1}$. Then $T=T_{1} \nearrow T_{2}$, where $X$ is the node labeled 2456 .

Suppose that $\stackrel{X}{T_{1}} \in \operatorname{DPT}\left(V_{1}\right)$ and $T_{2} \in \operatorname{DPT}\left(V_{2}\right)$, where $V_{1} \cap V_{2}=\emptyset$. If $X \in V_{2}$ and $\operatorname{rt}\left(T_{1}\right)<X$, then $T_{1} \nearrow_{X} T_{2} \in \operatorname{DPT}\left(V_{1} \cup V_{2}\right)$. In addition, every tree $T$ with more than one node can be written uniquely as $T_{1} \nearrow T_{2}$, by taking $T_{1}=T \mid \operatorname{rt}(T)^{(1)}$ and $T_{2}=T-T_{1}$.

Lemma 11.2. Let $k \in[2, n]$. Then

$$
d(n, k-1)=\sum_{a=1}^{k-1}\left(\begin{array}{c}
k-1 \\
a
\end{array}\right) d(a, a) d(n-a, k-a) .
$$

Proof. Let $T_{1}$ be a decreasing planar tree with nodes $A \subset[1, k-1],|A|=a<k$. There are $\left(\begin{array}{c}k-1 \\ a\end{array}\right) d(a, a)$ such trees. Meanwhile, let $T_{2}$ be a decreasing planar tree with nodes $[a+1, n]$ and largest leaf $L\left(T_{2}\right) \leq k$. Subtracting $a$ from the label of every node gives a bijection between such trees $T_{2}$ and the set $\operatorname{DPT}(n-a, k-a)$. Therefore the number of pairs $\left(T_{1}, T_{2}\right)$ is counted by the right-hand side of the desired equality. For each such pair, let

$$
f\left(T_{1}, T_{2}\right)=T_{1} \nearrow_{k} T_{2}
$$

The map $f$ is clearly injective, and $f\left(T_{1}, T_{2}\right) \in \operatorname{DPT}(n, k-1)$. On the other hand, for any $T \in \operatorname{DPT}(n, k-1)$, let $T^{\prime}=T \mid k^{(1)}$ and $T^{\prime \prime}=T-T^{\prime}$. Then $T^{\prime} \nearrow_{k} T^{\prime \prime}=T$. So $f$ maps onto $\operatorname{DPT}(n, k-1)$, and the desired equality follows. 
This formula may be taken as a recursive definition for the numbers $d(n, k)$. For small values of $n$ and $k$, they are given by the following table.

\begin{tabular}{c|cccccc} 
& $k=1$ & 2 & 3 & 4 & 5 & 6 \\
\hline$n=2$ & 1 & 1 & & & & \\
3 & 1 & 3 & 3 & & & \\
4 & 1 & 7 & 15 & 15 & & \\
5 & 1 & 15 & 57 & 105 & 105 & \\
6 & 1 & 31 & 105 & 561 & 945 & 945
\end{tabular}

Lemma 11.3. Let $1<k \leq n$. Then

$$
\begin{aligned}
\sum_{a=1}^{k-1} & \sum_{c=1}^{n-k-1}\left(\begin{array}{c}
k-1 \\
a
\end{array}\right)\left(\begin{array}{c}
n-k-1 \\
c
\end{array}\right) d(a+c, a) d(n-a-c, k-a)= \\
& \sum_{w=0}^{k-2} \sum_{y=1}^{n-k-1}\left(\begin{array}{c}
k-1 \\
w
\end{array}\right)\left(\begin{array}{c}
n-k-1 \\
y
\end{array}\right) d(w+y, w+1) d(n-w-y, k-w-1) .
\end{aligned}
$$

Proof. First, we interpret the sums on either side of the desired equality as enumerating certain kinds of decreasing planar trees. The left-hand side counts trees of the form $T=T^{\prime} \nearrow T^{\prime \prime} \in \operatorname{DPT}(n)$ such that

$$
\begin{aligned}
V\left(T^{\prime}\right) & =A \sqcup C, & L\left(T^{\prime}\right) & \in A, \\
V\left(T^{\prime \prime}\right) & =B \sqcup D \sqcup\{k, n\}, & L\left(T^{\prime \prime}\right) & \in B \sqcup\{k\},
\end{aligned}
$$

where

$$
\begin{array}{llrl}
A \sqcup B & =[1, k-1], & & 1 \leq a=|A| \leq k-1, \\
C \sqcup D=[k+1, n-1], & & 1 \leq c=|C| \leq n-k-1 .
\end{array}
$$

Meanwhile, the right-hand side counts trees of the form $U=U^{\prime} \nearrow U^{\prime \prime} \in \operatorname{DPT}(n)$ such that

$$
\begin{aligned}
V\left(U^{\prime}\right) & =W \sqcup Y, & L\left(T^{\prime}\right) & \in W \sqcup\{\min (Y)\}, \\
V\left(U^{\prime \prime}\right) & =X \sqcup Z \sqcup\{k, n\}, & L\left(T^{\prime \prime}\right) & \in X,
\end{aligned}
$$

where

$$
\begin{aligned}
W \sqcup X & =[1, k-1], & 0 \leq w & =|W| \leq k-2, \\
Y \sqcup Z & =[k+1, n-1], & 1 \leq y & =|Y| \leq n-k-1 .
\end{aligned}
$$

Second, we show how to perform "surgery" on a tree of the form (23) to obtain a tree of the form (24). Suppose that $T=T^{\prime} \nearrow T^{\prime \prime}$ satisfies (23). Define a tree $f(T)$ as follows.

- If $k$ has children in $T$, then $f(T)=T$. In this case $B \neq \emptyset$, so $A \subsetneq[1, k-1]$. Also, $L(T)<k$; in particular $L\left(T^{\prime \prime}\right) \in B$. Therefore, $T$ satisfies (24), with $W=A$, $X=B, Y=C, Z=D$.

- If $k$ is a leaf, then we form $f(T)$ by detaching all subtrees of $\min (C)$ and reattaching them to $k$ in the same birth order. Note that there is at least one such subtree since $\min (C)>L(T)$. Also, $L(f(T))=\min (C)$, and $f(T)$ satisfies (24), with

$$
\begin{aligned}
W & =A \backslash\{\text { descendants of } \min (C)\}, & & Y=C, \\
X & =B \sqcup\{\text { descendants of } \min (C)\}, & & Z=D .
\end{aligned}
$$

Third, we show how this surgery can be reversed. Suppose that $U=U^{\prime} \nearrow U^{\prime \prime}$ satisfies (24). Define a tree $g(U)$ as follows: 
- If $\min (Y)$ has children in $U$, then $g(U)=U$. In this case $W \neq \emptyset$. Also, $L(T)<k$; in particular $L\left(T^{\prime \prime}\right) \in X$. Therefore, $T$ satisfies (24), with $A=W$, $B=X, C=Y, D=Z$.

- If $\min (Y)$ is a leaf, then we form $g(U)$ by detaching all subtrees of $k$ and reattaching them to $\min (Y)$ in the same birth order. Note that since $k>L(U)$, this step is not trivial. Also, $L(g(U))=k$, and $g(U)$ satisfies (23), with

$$
\begin{array}{ll}
A=W \sqcup\{\text { descendants of } \min (Y)\}, & C=Y, \\
B=X \backslash\{\text { descendants of } \min (Y)\}, & D=Z .
\end{array}
$$

Finally, we show that the functions $f$ and $g$ are mutual inverses. Suppose that $T$ satisfies (23). In particular, $\min (C)$ has children in $T$, so if $f(T)=T$, then $g(f(T))=T$. On the other hand, if $k$ is a leaf in $T$, then by construction $\min (C)=$ $\min (Y)$ is a leaf in $f(T)$, and the two surgeries described above are inverses by definition. So $g(f(T))=T$ in any case. The proof that $f(g(U))=U$ is analogous. So $f$ and $g$ are bijections and we are done.

Lemma 11.4. Let $1<k \leq n$. Then

$$
d(n, k)=d(n-1, k)+\sum_{a=1}^{k-1} \sum_{c=0}^{n-k-1}\left(\begin{array}{c}
k \\
a
\end{array}\right)\left(\begin{array}{c}
n-k-1 \\
c
\end{array}\right) d(a+c, a) d(n-a-c, k-a) .
$$

Proof. Deleting the node $n$ gives a bijection between trees $T \in \operatorname{DPT}(n, k)$ such that $n$ has exactly one child, and $\operatorname{DPT}(n-1, k)$. This accounts for the term $d(n-1, k)$ on the right-hand side. On the other hand, suppose that $T \in \operatorname{DPT}(n, k)$ is a tree in which $n$ has more than one child. Then $T=T^{\prime} \nearrow T^{\prime \prime}$, where

$$
\begin{aligned}
V\left(T^{\prime}\right) & =A \sqcup C, & L\left(T^{\prime}\right) & \in A, \\
V\left(T^{\prime \prime}\right) & =B \sqcup D \sqcup\{n\}, & L\left(T^{\prime \prime}\right) & \in B, \\
A \sqcup B & =[1, k], & 1 \leq a & =|A| \leq k-1, \\
C \sqcup D & =[k+1, n-1], & 0 \leq c & =|C| \leq n-k-1 .
\end{aligned}
$$

Conversely, if a pair $\left(T^{\prime}, T^{\prime \prime}\right)$ satisfies these conditions, then $T=T^{\prime} \nearrow T^{\prime \prime}$ belongs to $\operatorname{DPT}(n, k)$, and $n$ has at least two children in $T$. These pairs are enumerated by the double sum on the right-hand side of the desired equality.

Applying Pascal's identity

$$
\left(\begin{array}{l}
k \\
a
\end{array}\right)=\left(\begin{array}{c}
k-1 \\
a
\end{array}\right)+\left(\begin{array}{l}
k-1 \\
a-1
\end{array}\right)
$$

to the equation of Lemma 11.4 we obtain

$$
\begin{aligned}
& d(n, k)=d(n-1, k) \\
& +\sum_{a=1}^{k-1} \sum_{c=0}^{n-k-1}\left(\begin{array}{c}
k-1 \\
a
\end{array}\right)\left(\begin{array}{c}
n-k-1 \\
c
\end{array}\right) d(a+c, a) d(n-a-c, k-a) \\
& +\sum_{a=1}^{k-1} \sum_{c=0}^{n-k-1}\left(\begin{array}{c}
k-1 \\
a-1
\end{array}\right)\left(\begin{array}{c}
n-k-1 \\
c
\end{array}\right) d(a+c, a) d(n-a-c, k-a) .
\end{aligned}
$$


Breaking off the $c=0$ term of the first double sum in (25) yields

$$
\begin{aligned}
& d(n, k)=d(n-1, k)+\sum_{a=1}^{k-1}\left(\begin{array}{c}
k-1 \\
a
\end{array}\right) d(a, a) d(n-a, k-a) \\
& +\sum_{a=1}^{k-1} \sum_{c=1}^{n-k-1}\left(\begin{array}{c}
k-1 \\
a
\end{array}\right)\left(\begin{array}{c}
n-k-1 \\
c
\end{array}\right) d(a+c, a) d(n-a-c, k-a) \\
& +\sum_{a=1}^{k-1} \sum_{c=0}^{n-k-1}\left(\begin{array}{c}
k-1 \\
a-1
\end{array}\right)\left(\begin{array}{c}
n-k-1 \\
c
\end{array}\right) d(a+c, a) d(n-a-c, k-a) .
\end{aligned}
$$

Applying Lemma 11.2 to the single sum and Lemma 11.3 to the first double sum in (26), we obtain

$$
\begin{aligned}
& d(n, k)=d(n-1, k)+d(n, k-1) \\
& +\sum_{w=0}^{k-2} \sum_{y=1}^{n-k-1}\left(\begin{array}{c}
k-1 \\
w
\end{array}\right)\left(\begin{array}{c}
n-k-1 \\
y
\end{array}\right) d(w+y, w+1) d(n-w-y, k-w-1) \\
& +\sum_{a=1}^{k-1} \sum_{c=0}^{n-k-1}\left(\begin{array}{c}
k-1 \\
a-1
\end{array}\right)\left(\begin{array}{c}
n-k-1 \\
c
\end{array}\right) d(a+c, a) d(n-a-c, k-a) .
\end{aligned}
$$

In the first double sum of [27], we may change the upper limit on $y$ from $n-k-1$ to $n-k$, because the additional summand is zero; in addition put $x=n-k-y$. In the second double sum, put $w=a-1$ and $x=n-k-1-c$. We obtain

$$
\begin{aligned}
& d(n, k)=d(n-1, k)+d(n, k-1) \\
& +\sum_{w=0}^{k-2} \sum_{x=0}^{n-k-1}\left(\begin{array}{c}
k-1 \\
w
\end{array}\right)\left(\begin{array}{c}
n-k-1 \\
x-1
\end{array}\right) d(n-k+w-x, w+1) d(k-w+x, k-w-1) \\
& +\sum_{w=0}^{k-2} \sum_{x=0}^{n-k-1}\left(\begin{array}{c}
k-1 \\
w
\end{array}\right)\left(\begin{array}{c}
n-k-1 \\
x
\end{array}\right) d(n-k+w-x, w+1) d(k-w+x, k-w-1)
\end{aligned}
$$

Now, another application of Pascal's identity, together with the base cases mentioned previously, yields the following:

Proposition 11.5. The numbers $d(n, k)$ satisfy the recurrence

$$
\begin{aligned}
& d(n, k)=d(n-1, k)+d(n, k-1) \\
& +\sum_{w=0}^{k-2} \sum_{x=0}^{n-k-1}\left(\begin{array}{c}
k-1 \\
w
\end{array}\right)\left(\begin{array}{c}
n-k \\
x
\end{array}\right) d(n-k+w-x, w+1) d(k-w+x, k-w-1) .
\end{aligned}
$$

Finally, we come to the geometric reason for all this enumeration. The recurrence given by Proposition 11.5 is easily turned into one giving the lower bounds for the degree of the flattened slope variety $\tilde{\mathcal{S}}(n, k)$, as we now show.

Theorem 11.6. We have $\operatorname{deg} \tilde{\mathcal{S}}(n, k) \geq d(n-1, n-k)$. In particular,

$$
\operatorname{deg} \tilde{\mathcal{S}}\left(K_{n}\right) \geq d(n-1, n-1)=|\operatorname{DPT}(n-1)| .
$$


Proof. Replacing $d(i, j)$ with $e(i+1, i-j+1)$ in Proposition 11.5 we obtain

$$
\begin{aligned}
& e(n+1, n-k+1)=e(n, n-k)+e(n+1, n-k+2) \\
& +\sum_{w=0}^{k-2} \sum_{x=0}^{n-k-1}\left(\begin{array}{c}
k-1 \\
w
\end{array}\right)\left(\begin{array}{c}
n-k \\
x
\end{array}\right) \\
& \quad \times e(n-k+w-x+1, n-k-x) e(k-w+x+1, x+2) .
\end{aligned}
$$

Now, setting

$$
n=n^{\prime}-1, \quad k=n^{\prime}-k^{\prime}, \quad x=k^{\prime}-w^{\prime}-1, \quad w=x^{\prime}
$$

and removing the primes from the new variables, we obtain the recurrence of Theorem 10.4 It follows that $d(n, k)=e(n+1, n-k+1)$ for all $n$ and $k$, or equivalently $e(n, k)=d(n-1, n-k)$.

\section{Proof of the Main Theorem}

With all the pieces in place, we can now prove our main result.

Proof of Theorem 1.1. The variety $\tilde{\mathcal{S}}\left(K_{n}\right)$ is irreducible and reduced, hence is defined scheme-theoretically by the prime ideal $\sqrt{I_{n}}$. In addition, we have

$$
J_{n} \subset \operatorname{in}\left(I_{n}\right) \subset \operatorname{in}\left(\sqrt{I_{n}}\right) .
$$

By Theorem 5.2 we have

$$
\operatorname{codim} J_{n}=2 n-3=\operatorname{dim} \tilde{\mathcal{S}}\left(K_{n}\right)=\operatorname{codim} \sqrt{I_{n}}
$$

and by Theorem 7.4 and Theorem 11.6 we have

$$
\operatorname{deg} J_{n}=\frac{(2 n-4) !}{2^{n-2}(n-2) !} \leq \operatorname{deg} \sqrt{I_{n}} .
$$

We have seen in Theorem 8.3 that the Stanley-Reisner complex of $J_{n}$ is shellable. Hence $J_{n}$ is Cohen-Macaulay [3] Theorem 5.1.13]. In particular it is unmixed, so any strictly larger ideal has either larger codimension or smaller degree. Meanwhile, $\operatorname{in}\left(\sqrt{I_{n}}\right)$ has the same codimension and degree as $\operatorname{in}\left(\sqrt{I_{n}}\right)$ by [5] Theorem 15.26], Therefore equality holds in (29). Since $J_{n}=\operatorname{in}\left(I_{n}\right)$ is Cohen-Macaulay, so is $I_{n}$ 14, Corollary B.2.1]. It follows that $\tilde{\mathcal{S}}\left(K_{n}\right)$ is Cohen-Macaulay. Finally, the formula for the Hilbert series is a restatement of the results of Section 9

We remark that for the degree bounds in Theorem 11.6 to be sharp, equality must hold in the lower bounds of Theorem 10.4 That is, the flattened slope variety $\tilde{\mathcal{S}}(n, k)$ has degree exactly $d(n-1, n-k)$.

We conclude by mentioning some problems for further study. Using the computer algebra system Macaulay [1, we have verified that $I_{n}$ is generated by the (cubic) tree polynomials of the $\left(\begin{array}{c}n \\ 4\end{array}\right)$ copies of $K_{4}$ arising as subgraphs of $K_{n}$, for $n \leq$ 9. Accordingly, we conjecture that they do so for all $n$. The difficulty is that there appears to be no canonical way to write a tree polynomial $\tau(W)$ as an $R_{K_{n}}$ linear combination of the $\tau\left(K_{4}\right)$ 's, where $W$ is a wheel with five or more vertices. Further computations using Macaulay suggest that the wheel polynomials may form a universal Gröbner basis for $I_{n}$ (that is, a Gröbner basis with respect to every term ordering), but it is not clear how to prove this.

The technique of using the Stanley-Reisner complex to obtain information about graph varieties may be applicable to graphs other than $K_{n}$. The likeliest candidates 
are graphs with many symmetries, such as threshold graphs or complete bipartite graphs. The degree and Hilbert series of the slope variety of a graph are combinatorial invariants for which it would be nice to have a more elementary graph-theoretic description. In addition, it would be of interest to determine whether $\tilde{\mathcal{S}}(G)$ is always defined scheme-theoretically by the tree polynomials of rigidity circuit subgraphs of $G$, and whether it is always Cohen-Macaulay. (We have computed $I_{G}$ for several small graphs; in all cases the Cohen-Macaulay property is satisfied.)

An anonymous referee has pointed out that the space $\mathscr{T}_{n}$ of phylogenetic trees on $n$ vertices, constructed by Billera, Holmes and Vogtmann in [2], has degree $(2 n-4) ! /\left(2^{n-2}(n-2) !\right)$, the same as that of $\tilde{\mathcal{S}}\left(K_{n}\right)$. Moreover, for $0<m<n$, the space $\mathscr{T}_{n}$ contains many subspaces isomorphic to $\mathscr{T}_{m}$ [2] p. 743], just as $\tilde{\mathcal{S}}\left(K_{n}\right)$ contains many copies of $\tilde{\mathcal{S}}\left(K_{m}\right)$ (see Section 10). It would be interesting to explore possible connections between pictures and phylogenetic trees.

Other spaces related to graph varieties include the Fulton-Macpherson compactification of configuration space [6] and the De Concini-Procesi wonderful model of subspace arrangements [4. The results of this article might serve as a starting point for studying these relationships in more detail.

\section{REFERENCES}

[1] David Bayer and Michael Stillman. Macaulay: A computer algebra system for algebraic geometry. Software, 1994. Available at http://www.math.columbia.edu/ bayer/Macaulay/.

[2] Louis J. Billera, Susan P. Holmes, and Karen Vogtmann. Geometry of the space of phylogenetic trees. Adv. in Appl. Math., 27(4):733-767, 2001.

[3] Winfried Bruns and Jürgen Herzog. Cohen-Macaulay rings. Cambridge University Press, Cambridge, 1993

[4] Corrado De Concini and Claudio Procesi. Wonderful models of subspace arrangements. Selecta Math. (N.S.), 1(3):459-494, 1995.

[5] David Eisenbud. Commutative algebra with a view toward Algebraic Geometry. SpringerVerlag, New York, 1995.

[6] William Fulton and Robert MacPherson. A compactification of configuration spaces. Ann. of Math. (2), 139(1):183-225, 1994.

[7] Jack Graver, Brigitte Servatius, and Herman Servatius. Combinatorial rigidity. American Mathematical Society, Providence, RI, 1993.

[8] Germain Kreweras and Yves Poupard. Sur les partitions en paires d'un ensemble fini totalement ordonné. Pub. Inst. Stat. Univ. Paris, XXIII, fasc. 1-2:57-74, 1978.

[9] Jeremy L. Martin. Graph Varieties. PhD thesis, University of California, San Diego, 2002.

[10] Jeremy L. Martin. Geometry of graph varieties. Trans. Amer. Math. Soc., 355(10):4151-4169, 2003.

[11] Nicholas J.A. Sloane. The On-Line Encyclopedia of Integer Sequences, 2002. Published elecronically at http://www.research.att.com/ njas/sequences/.

[12] Richard P. Stanley. Combinatorics and commutative algebra. Birkhäuser Boston Inc., Boston, MA, second edition, 1996.

[13] Richard P. Stanley. Enumerative combinatorics. Vol. 2. Cambridge University Press, Cambridge, 1999.

[14] Wolmer V. Vasconcelos. Computational methods in commutative algebra and algebraic geometry. Springer-Verlag, Berlin, 1998.

[15] Douglas B. West. Introduction to graph theory. Prentice Hall Inc., Upper Saddle River, NJ, second edition, 2001.

Department of Mathematics, University of California, San Diego, La Jolla, CA 92093-0112

Current address: Department of Mathematics, University of Kansas, Lawrence, KS 66044

E-mail address: jmartin@math.ku.edu 